\title{
On the computational aspects of a symmetric multidomain boundary element method approach for elastoplastic analysis
}

\author{
L Zito, E Parlavecchio, and T Panzeca* \\ Dipartimento di Ingegneria Strutturale, Aerospaziale e Geotecnica, Università di Palermo, Palermo, Italy
}

The manuscript was received on 18 March 2010 and was accepted after revision for publication on 22 September 2010.

DOI: $10.1243 / 03093247 J S A 651$

\begin{abstract}
The symmetric boundary element method (SBEM) is applied to the elasto-plastic analysis of bodies subdivided into substructures. This methodology is based on the use of: a multidomain SBEM approach, for the evaluation of the elastic predictor; a return mapping algorithm based on the extremal paths theory, for the evaluation of inelastic quantities characterizing the plastic behaviour of each substructure; and a transformation of the domain inelastic integrals of each substructure into corresponding boundary integrals. The elastic analysis is performed by using the SBEM displacement approach, which has the advantage of creating system equations that only consist of nodal kinematical unknowns at the interface boundary among substructures. The elastoplastic solution utilizes a strain-driven strategy that is characterized by the evaluation of the elastic predictor that is a function of the initial conditions and the load increment. The predictor phase is followed by the use of a return mapping algorithm defined by introducing the extremal paths theory to remove the time integration. Then the computed plastic strains are considered to be constant inelastic actions imposed inside the substructure in a step-by-step procedure. Their presence involves domain integrals with singular kernels. These integrals are considered as Cauchy principal values with which the related free term is associated. In order to compute these domain integrals, the radial integral method is applied to remove the strong singularity.
\end{abstract}

Keywords: elastoplasticity, symmetric boundary element method, multidomain approach, singular domain integral, return mapping algorithm

\section{INTRODUCTION}

This paper presents an efficient methodology that uses the symmetric boundary element method (SBEM) for the elasto-plastic analysis of multidomain-type problems $[\mathbf{1}, \mathbf{2}]$. The results of this method are extremely advantageous and competitive for non-linear problems, not only because the analysis can be performed on large subdomains but also because only a small number of BEM-elements (hereafter bem-es) are required. The latter is due to the computation in closed form of all the solving system coefficients, which involves no numerical instability in the solution.

*Corresponding author: Dipartimento di Ingegneria Strutturale, Aerospaziale e Geotecnica, Università di Palermo, viale delle Scienze, Palermo 90128, Italy.

email: tpanzeca@tiscali.it
The application of the BEM to inelastic problems, in its collocation version, may be found in Brebbia et al. [3] and in Aliabadi [4], and the references therein. Implicit approaches for the solution of nonlinear problems were first introduced by Telles and Carrer [5]. They have two important features: a reduced number of iterations and an increased computational effort for each load increment. Banerjee et al. [6] presented a variable stiffness formulation for such problems. Bonnet and Mukherjee [7], in solving non-linear problems, have introduced an implicit BEM scheme that uses a sensitivity analysis with a local consistent tangent operator (CTO). This method, which was first proposed by Simo and Taylor [8] in the finite element method (FEM) context, exploits the quadratic rate of convergence which can be achieved by utilizing consistent elasto-plastic constitutive relations in the Newton-Raphson iterative process. Paulino and Yong [9] have presented an 
assessment and comparison of BEM formulations for elasto-plasticity using the CTO and the continuum tangent operator. Recently, Mallardo and Alessandri [10] developed an arc-length procedure combined with the BEM for use in plasticity and damage studies.

A Galerkin approach to boundary element elastoplastic analysis has been proposed by Maier and Polizzotto [11], Polizzotto [12, 13], and Lie et al. [14] by using integral equations of the linear elasticity in terms of generalized quantities. However, because of the known computational difficulties, there are very few numerical applications, and this approach tends to be applied only to simple examples.

The present paper describes a more general approach that couples the SBEM with a return mapping scheme for application to non-linear problems. Particular attention is paid to the case of two-dimensional (2D) continuous solids so that the elasticperfectly-plastic von Mises' law and the associated plastic flow can be applied to simplify the derivation of the equations. The bem-es are introduced in regions of potential non-linear behaviour and the multidomain displacements approach, developed by Panzeca et al. [15] and Panzeca et al. [16], is used in the non-linear analysis.

This approach is characterized by a strong condensation of the mechanical and kinematical nodal variables of the bem-es, which allows the evaluation in a subsequent phase of all the domain quantities, as tractions and stresses, displacements, and strains, through use of Somigliana identities (SIs). It is similar to the FEM but has several advantages created by the use of the SBEM approach including:

(a) domain discretization into a very small number of bem-es is only performed in the zones in which the plastic phenomenon takes place, the elastic zone of the body is treated as a unique bem-e;

(b) the computation of all solution system coefficients in closed form involves a perfectly stable solution vector whatever the geometry and the dimension of the bem-e;

(c) the use of symmetric algebraic operators in the non-linear analysis;

(d) the equilibrium and compatibility are ensured at every point inside the domain because of the use of SIs, whereas those on the interface boundary are treated in terms of generalized variables;

(e) a considerable reduction in the number of unknowns.

This approach permits the development of an analysis of a body subjected to boundary and domain actions, both elastic and inelastic.
One of the fundamental features of the implementation of plasticity models is the numerical integration of the constitutive equations. This integration is carried out locally at each Gauss point in a typical FEM or BEM implementation with return mapping algorithms being a standard strategy. Such algorithms consist of a first phase in which the predictor or trial stress is obtained by using an elastic analysis and a second phase in which the predictor is relaxed onto the yield surface by iteratively generating and modifying plastic strain components by increments. With regard to this method, Ortiz and Simo [17] proposed a new class of integration algorithms that use the operator splitting method. These algorithms are applicable to a general class of plastic and visco-plastic constitutive models, i.e. with hardening and softening behaviour and with associated or non-associated flow rules. Ortiz and Martin [18] and, recently, Bilotta and Casciaro [19] have formulated elasto-plastic problems in which the constitutive relations are exactly integrated along particular paths in accordance to the theory proposed by Ponter and Martin [20]. In the present formulation, which uses the SBEM, this strategy is recast as an iterative procedure in which the plastic strains are considered to be constant inelastic actions imposed by the bem-e. Their presence involves domain integrals with singular kernels. These integrals are considered as Cauchy principal values (CPVs) to which the Bui free term [21] can be associated. Therefore, regularization techniques may be applied to cut-off the strong singularity by using the radial integral method (RIM) [22] to transform the domain integrals into boundary integrals for each bem-e.

Also, generalized boundary tractions have to be introduced to be able to use the SBEM. Presented here is new procedure for the evaluation of the boundary traction [23] due to plastic actions that is different to the methods proposed in references [24] and [25] where the stress boundary integral equation for points on the boundaries including the corners are dealt with using a collocation approach. In this paper, the traction on the boundary, caused by plastic strain, is obtained trough a limit operation and the integral by virtue of having a strong singularity can be defined as a CPV to which the Bui free term and an additional free term that depends on the boundary geometry are added.

An algorithm has been implemented using the Karnak.sGbem code [26] and numerical tests have been performed to show the computational performance of the method. 


\section{ANALYSIS OF A MULTIDOMAIN USING A DISPLACEMENT APPROACH}

In this section, an elastic analysis is performed that uses the SBEM displacements approach developed by Panzeca et al. [15] and Panzeca et al. [16]. A general strategy that defines the algebraic operators in terms of the geometry and the boundary typology for each bem-e is presented. This strategy has the advantage of obtaining a system that has quantities that are solely related to the interface boundaries and it is therefore computationally simpler than other formulations based on FEM or BEM strategies.

\subsection{The elasticity relations for each bem-e}

Consider a 2D body with a domain $\Omega$ and boundary $\Gamma$, referred to a Cartesian system $(o, x, y)$, subjected to forces $\overline{\boldsymbol{f}}$ on the free boundary $\Gamma_{2}$, to displacements $\overline{\boldsymbol{u}}$ imposed on constrained boundary $\Gamma_{1}$, and to body forces $\overline{\boldsymbol{b}}$ and inelastic actions $\overline{\boldsymbol{\varepsilon}}^{\mathrm{P}}$ in $\Omega$.

The physical and geometrical characteristics are zone-wise variables. The approach of this paper is to obtain the elastic response in terms of the displacements $\boldsymbol{u}_{2}$ on $\Gamma_{2}$, reactive forces $\boldsymbol{f}_{1}$ on $\Gamma_{1}$, and the displacements, strains, and stresses in $\Omega$ by using the displacement approach of the SBEM.

As a first step, an appropriate subdivision of the domain into bem-es, each having constant physical and geometrical characteristics, is performed. This subdivision involves the introduction of interface boundaries $\Gamma_{0}$ between contiguous bem-es and, as a consequence, the creation of two new unknown quantities in the analysis problem, i.e. the displacement vector $\boldsymbol{u}_{0}$ and the interface traction vector $\boldsymbol{t}_{0}$.

For each bem-e it is necessary to determine an elasticity equation that connects the mechanical quantities to the kinematical ones, both associated to the interface zones. Consider the following Dirichlet- Neumann conditions

$$
\begin{aligned}
& \boldsymbol{u}_{1}=\overline{\boldsymbol{u}}_{1} \text { on } \Gamma_{1} \\
& \boldsymbol{t}_{2}=\overline{\boldsymbol{f}}_{2} \text { on } \Gamma_{2}
\end{aligned}
$$

If the SIs of the displacements and tractions are introduced into equations (1a) and (1b), then the following boundary integral equations may be written, where $\boldsymbol{f}_{0}$ is the vector that characterizes the layered force distribution acting on $\Gamma_{0}$

$$
\begin{aligned}
& \boldsymbol{u}_{1}\left(\boldsymbol{f}_{1},-\boldsymbol{u}_{2}, \boldsymbol{f}_{0},-\boldsymbol{u}_{0}\right)+\boldsymbol{u}_{1}\left(\overline{\boldsymbol{f}}_{2},-\overline{\boldsymbol{u}}_{1}, \overline{\boldsymbol{b}}\right) \\
& \quad+\boldsymbol{u}_{1}\left(\overline{\boldsymbol{\varepsilon}}^{\mathrm{p}}\right)=\overline{\boldsymbol{u}}_{1}
\end{aligned}
$$

$$
\begin{aligned}
& \boldsymbol{t}_{2}\left(\boldsymbol{f}_{1},-\boldsymbol{u}_{2}, \boldsymbol{f}_{0},-\boldsymbol{u}_{0}\right)+\boldsymbol{t}_{2}\left(\overline{\boldsymbol{f}}_{2},-\overline{\boldsymbol{u}}_{1}, \overline{\boldsymbol{b}}\right) \\
& \quad+\boldsymbol{t}_{2}\left(\overline{\boldsymbol{\varepsilon}}^{\mathrm{p}}\right)=\overline{\boldsymbol{f}}_{2}
\end{aligned}
$$

The unknown $\boldsymbol{u}_{0}$ and $\boldsymbol{t}_{0}$ vectors on $\Gamma_{0}$ may be written as

$$
\begin{aligned}
\boldsymbol{u}_{0}= & \boldsymbol{u}_{0}\left(\boldsymbol{f}_{1},-\boldsymbol{u}_{2}, \boldsymbol{f}_{0},-\boldsymbol{u}_{0}\right) \\
& +\boldsymbol{u}_{0}\left(\overline{\boldsymbol{f}}_{2},-\overline{\boldsymbol{u}}_{1}, \overline{\boldsymbol{b}}\right)+\boldsymbol{u}_{0}\left(\overline{\boldsymbol{\varepsilon}}^{\mathrm{p}}\right) \\
\boldsymbol{t}_{0}= & \boldsymbol{t}_{0}\left(\boldsymbol{f}_{1},-\boldsymbol{u}_{2}, \boldsymbol{f}_{0},-\boldsymbol{u}_{0}\right) \\
& +\boldsymbol{t}_{0}\left(\overline{\boldsymbol{f}}_{2},-\overline{\boldsymbol{u}}_{1}, \overline{\boldsymbol{b}}\right)+\boldsymbol{t}_{0}\left(\overline{\boldsymbol{\varepsilon}}^{\mathrm{p}}\right)
\end{aligned}
$$

The equations (2a), (2b), and (3a) have to be rewritten in a different way while equation (3b) remains unchanged.

$$
\begin{aligned}
& \boldsymbol{u}_{1}\left(\boldsymbol{f}_{1},-\boldsymbol{u}_{2}, \boldsymbol{f}_{0},-\boldsymbol{u}_{0}\right)+\boldsymbol{u}_{1}\left(\overline{\boldsymbol{f}}_{2},-\overline{\boldsymbol{u}}_{1}, \overline{\boldsymbol{b}}\right)+\boldsymbol{u}_{1}\left(\overline{\boldsymbol{\varepsilon}}^{\mathrm{p}}\right)=\boldsymbol{0} \\
& \boldsymbol{t}_{2}\left(\boldsymbol{f}_{1},-\boldsymbol{u}_{2}, \boldsymbol{f}_{0},-\boldsymbol{u}_{0}\right)+\boldsymbol{t}_{2}\left(\overline{\boldsymbol{f}}_{2},-\overline{\boldsymbol{u}}_{1}, \overline{\boldsymbol{b}}\right)+\boldsymbol{t}_{2}\left(\overline{\boldsymbol{\varepsilon}}^{\mathrm{p}}\right)=\boldsymbol{0} \\
& \boldsymbol{u}_{0}\left(\boldsymbol{f}_{1},-\boldsymbol{u}_{2}, \boldsymbol{f}_{0},-\boldsymbol{u}_{0}\right)+\boldsymbol{u}_{0}\left(\overline{\boldsymbol{f}}_{2},-\overline{\boldsymbol{u}}_{1}, \overline{\boldsymbol{b}}\right)+\boldsymbol{u}_{0}\left(\overline{\boldsymbol{\varepsilon}}^{\mathrm{p}}\right)=\boldsymbol{0} \\
& \boldsymbol{t}_{0}=\boldsymbol{t}_{0}\left(\boldsymbol{f}_{1},-\boldsymbol{u}_{2}, \boldsymbol{f}_{0},-\boldsymbol{u}_{0}\right)+\boldsymbol{t}_{0}\left(\overline{\boldsymbol{f}}_{2},-\overline{\boldsymbol{u}}_{1}, \overline{\boldsymbol{b}}\right)+\boldsymbol{t}_{0}\left(\overline{\boldsymbol{\varepsilon}}^{\mathrm{p}}\right)
\end{aligned}
$$

The plastic strains vector $\overline{\boldsymbol{\varepsilon}}^{\mathrm{P}}$ is obtainable through an evaluation strategy defined in the next section.

Boundary discretization is introduced into the boundary elements by making the following modelling for all the known and unknown quantities

$$
\begin{gathered}
\boldsymbol{f}=\boldsymbol{\Psi}_{f} \boldsymbol{F} \\
\boldsymbol{u}=\boldsymbol{\Psi}_{u} \boldsymbol{U}
\end{gathered}
$$

where $\boldsymbol{F}$ and $\boldsymbol{U}$ indicate the nodal value vectors of the reactions $\left(\boldsymbol{F}_{1}\right.$ on $\Gamma_{1}$ and $\boldsymbol{F}_{0}$ on $\left.\Gamma_{0}\right)$ and of the displacements $\left(\boldsymbol{U}_{2}\right.$ on $\Gamma_{2}$ and $\boldsymbol{U}_{0}$ on $\left.\Gamma_{0}\right)$ defined at the boundary nodes.

The weighting of all the coefficients of equations (4a) to (4d) must now be performed. The weighting is performed in an energetically dual way based on the Galerkin approach: the coefficients defining displacements (or tractions) are weighted through the shape functions modelling the forces (or displacements). In this way it is possible to obtain the following block system 


$$
\begin{aligned}
\left|\begin{array}{c}
\boldsymbol{\boldsymbol { O }} \\
\boldsymbol{\boldsymbol { O }} \\
\boldsymbol{\boldsymbol { \sigma }} \\
\boldsymbol{P}_{0}
\end{array}\right| & =\left|\begin{array}{ccc:c}
\mathbf{A}_{\mathrm{u} 1, \mathrm{u} 1} & \mathbf{A}_{\mathrm{u} 1, \mathrm{f} 2} & \mathbf{A}_{\mathrm{u} 1, \mathrm{u} 0} & \mathbf{A}_{\mathrm{u} 1, \mathrm{f} 0} \\
\mathbf{A}_{\mathrm{f} 2, \mathrm{u} 1} & \mathbf{A}_{\mathrm{f} 2, \mathrm{f} 2} & \mathbf{A}_{\mathrm{f} 2, \mathrm{u} 0} & \mathbf{A}_{\mathrm{f} 2 \mathrm{f} 0} \\
\mathbf{A}_{\mathrm{u} 0, \mathrm{u} 1} & \mathbf{A}_{\mathrm{u} 0, \mathrm{f} 2} & \mathbf{A}_{\mathrm{u} 0, \mathrm{u} 0} & \overline{\mathbf{A}}_{\mathrm{u} 0, \mathrm{f} 0} \\
\hdashline \mathbf{A}_{\mathrm{f} 0, \mathrm{u} 1} & \mathbf{A}_{\mathrm{f} 0, \mathrm{f} 2} & \overline{\mathbf{A}}_{\mathrm{f} 0, \mathrm{u} 0} & \mathbf{A}_{\mathrm{f} 0, \mathrm{f} 0}
\end{array}\right|\left|\begin{array}{c}
\boldsymbol{F}_{1} \\
-\boldsymbol{U}_{2} \\
\boldsymbol{F}_{0} \\
-\boldsymbol{U}_{0}
\end{array}\right| \\
& +\left|\begin{array}{c}
\hat{\boldsymbol{W}}_{1}^{\mathrm{e}} \\
\hat{\boldsymbol{P}}_{2}^{\mathrm{e}} \\
\hat{\boldsymbol{W}}_{0}^{\mathrm{e}} \\
\hat{\boldsymbol{L}}_{0}^{\mathrm{e}}
\end{array}\right|+\left|\begin{array}{c}
\hat{\boldsymbol{W}}_{1}^{\mathrm{p}} \\
\hat{\boldsymbol{P}}_{2}^{\mathrm{p}} \\
\hat{\boldsymbol{W}}_{0}^{\mathrm{p}} \\
\hat{\boldsymbol{L}}_{0}^{\mathrm{p}}
\end{array}\right|
\end{aligned}
$$

In this equation, the first two conditions $\int_{\Gamma_{1}} \boldsymbol{\psi}_{f}^{\mathrm{T}}\left(\boldsymbol{u}_{1}-\right.$ $\left.\overline{\boldsymbol{u}}_{1}\right)=0$ and $\int_{\Gamma_{2}} \boldsymbol{\psi}_{u}^{\mathrm{T}}\left(\boldsymbol{t}_{2}-\overline{\boldsymbol{f}}_{2}\right)=0$ may be considered as Dirichlet-Neumann conditions in weighted form. The other two expressions weight the displacements and tractions at the interface zones. In particular, the weighting of equation (4d) (i.e. $P_{0}=\int_{\Gamma_{0}} \psi_{u}^{\mathrm{T}} \boldsymbol{t}_{0}$ ) collects the values of the generalized tractions defined on the boundary elements of $\Gamma_{0}$.

In equation (6), the following conditions have been set for the matrix coefficients

$$
\begin{aligned}
& \mathbf{A}_{u 1, u 1}=\int_{\Gamma_{1}} \boldsymbol{\psi}_{f}^{\mathrm{T}} \int_{\Gamma_{1}} \mathbf{G}_{u u} \boldsymbol{\psi}_{f}=\mathbf{A}_{u 1, u 1}^{\mathrm{T}}, \\
& \mathbf{A}_{u 1, f 2}=\int_{\Gamma_{1}} \boldsymbol{\psi}_{f}^{\mathrm{T}} \int_{\Gamma_{2}} \mathbf{G}_{u t} \boldsymbol{\psi}_{u}=\mathbf{A}_{f 2, u 1}^{\mathrm{T}}, \\
& \mathbf{A}_{f 2, f 2}=\int_{\Gamma_{2}} \boldsymbol{\psi}_{u}^{\mathrm{T}} \int_{\Gamma_{2}} \mathbf{G}_{t t} \boldsymbol{\psi}_{u}=\mathbf{A}_{f 2, f 2}^{\mathrm{T}} \\
& \mathbf{A}_{u 1, u 0}=\int_{\Gamma_{1}} \boldsymbol{\psi}_{f}^{\mathrm{T}} \int_{\Gamma_{0}} \mathbf{G}_{u u} \boldsymbol{\psi}_{f}=\mathbf{A}_{u 0, u 1}^{\mathrm{T}}, \\
& \mathbf{A}_{f 2, u 0}=\int_{\Gamma_{2}} \boldsymbol{\psi}_{u}^{\mathrm{T}} \int_{\Gamma_{0}} \mathbf{G}_{t u} \boldsymbol{\psi}_{f}=\mathbf{A}_{u 0, f 2}^{\mathrm{T}}, \\
& \mathbf{A}_{u 1, u 0}=\int_{\Gamma_{0}} \boldsymbol{\psi}_{f}^{\mathrm{T}} \int_{\Gamma_{0}} \mathbf{G}_{u u} \boldsymbol{\psi}_{f}=\int_{\Gamma_{1}} \boldsymbol{A}_{f 0, u 0}^{\mathrm{T}} \int_{\Gamma_{2}} \mathbf{G}_{t u} \boldsymbol{\psi}_{f}=\mathbf{A}_{f 0, u 1}^{\mathrm{T}}, \\
& \mathbf{A}_{f 2, f 0}=\int_{\Gamma_{2}} \boldsymbol{\psi}_{u}^{\mathrm{T}} \int_{\Gamma_{0}} \mathbf{G}_{t t} \boldsymbol{\psi}_{u}=\mathbf{A}_{f 0, f 2}^{\mathrm{T}}, \\
& \mathbf{A}_{f 0, f 0}=\int_{\Gamma_{0}} \boldsymbol{\psi}_{u}^{\mathrm{T}} \int_{\Gamma_{0}} \mathbf{G}_{t t} \boldsymbol{\psi}_{u}=\mathbf{A}_{f 0, f 0}^{\mathrm{T}} \\
& \overline{\mathbf{A}}_{u 0, f 0}=\int_{\Gamma_{0}} \boldsymbol{\psi}_{f}^{\mathrm{T}} \oint_{\Gamma_{0}} \mathbf{G}_{u t} \boldsymbol{\psi}_{u}+\frac{1}{2} \int_{\Gamma_{0}} \boldsymbol{\psi}_{f}^{\mathrm{T}} \boldsymbol{\psi}_{u}=\overline{\mathbf{A}}_{f 0, u 0}^{\mathrm{T}}
\end{aligned}
$$

for the elastic load vector

$$
\begin{aligned}
\hat{\boldsymbol{W}}_{1}^{\mathrm{e}}= & \int_{\Gamma_{1}} \boldsymbol{\psi}_{f}^{\mathrm{T}} \oint_{\Gamma_{1}} \mathbf{G}_{u t} \boldsymbol{\psi}_{u}\left(-\overline{\boldsymbol{U}}_{1}\right) \\
& +\frac{1}{2} \int_{\Gamma_{1}} \boldsymbol{\psi}_{f}^{\mathrm{T}} \boldsymbol{\psi}_{u}\left(-\overline{\boldsymbol{U}}_{1}\right)+\int_{\Gamma_{1}} \boldsymbol{\psi}_{f}^{\mathrm{T}} \int_{\Gamma_{2}} \mathbf{G}_{u u} \boldsymbol{\psi}_{f} \overline{\boldsymbol{F}}_{2} \\
& +\int_{\Gamma_{1}} \boldsymbol{\psi}_{f}^{\mathrm{T}} \int_{\Omega} \mathbf{G}_{u u} \overline{\boldsymbol{b}}
\end{aligned}
$$$$
\hat{\boldsymbol{P}}_{2}^{\mathrm{e}}=\int_{\Gamma_{2}} \boldsymbol{\psi}_{u}^{\mathrm{T}} \int_{\Gamma_{1}} \mathbf{G}_{t t} \boldsymbol{\psi}_{u}\left(-\overline{\boldsymbol{U}}_{1}\right)+\int_{\Gamma_{2}} \boldsymbol{\psi}_{u}^{\mathrm{T}} \oint_{\Gamma_{2}} \mathbf{G}_{t u} \boldsymbol{\psi}_{f} \overline{\boldsymbol{F}}_{2}
$$$$
-\frac{1}{2} \int_{\Gamma_{2}} \boldsymbol{\psi}_{u}^{\mathrm{T}} \boldsymbol{\psi}_{f} \overline{\boldsymbol{F}}_{2}+\int_{\Gamma_{2}} \boldsymbol{\psi}_{u}^{\mathrm{T}} \int_{\Omega} \mathbf{G}_{t u} \overline{\boldsymbol{b}}
$$

$$
\begin{aligned}
\hat{\boldsymbol{W}}_{0}^{\mathrm{e}}= & \int_{\Gamma_{0}} \boldsymbol{\psi}_{f}^{\mathrm{T}} \int_{\Gamma_{1}} \mathbf{G}_{u t} \boldsymbol{\psi}_{u}\left(-\overline{\boldsymbol{U}}_{1}\right) \\
& +\int_{\Gamma_{0}} \boldsymbol{\psi}_{f}^{\mathrm{T}} \int_{\Gamma_{2}} \mathbf{G}_{u u} \boldsymbol{\psi}_{f} \overline{\boldsymbol{F}}_{2}+\int_{\Gamma_{0}} \boldsymbol{\psi}_{f}^{\mathrm{T}} \int_{\Omega} \mathbf{G}_{u u} \overline{\boldsymbol{b}}
\end{aligned}
$$

$$
\begin{aligned}
\hat{\boldsymbol{L}}_{0}^{\mathrm{e}}= & \int_{\Gamma_{0}} \boldsymbol{\psi}_{u}^{\mathrm{T}} \int_{\Gamma_{1}} \mathbf{G}_{t t} \boldsymbol{\psi}_{u}\left(-\overline{\boldsymbol{U}}_{1}\right) \\
& +\int_{\Gamma_{0}} \boldsymbol{\psi}_{u}^{\mathrm{T}} \int_{\Gamma_{2}} \mathbf{G}_{t u} \boldsymbol{\psi}_{f} \overline{\boldsymbol{F}}_{2}+\int_{\Gamma_{0}} \boldsymbol{\psi}_{u}^{\mathrm{T}} \int_{\Omega} \mathbf{G}_{t u} \overline{\boldsymbol{b}}
\end{aligned}
$$

and for the plastic load vector

$$
\begin{aligned}
& \hat{\boldsymbol{W}}_{1}^{\mathrm{p}}=\int_{\Gamma_{1}} \boldsymbol{\psi}_{f}^{\mathrm{T}} \int_{\Omega} \mathbf{G}_{u \sigma} \overline{\boldsymbol{\varepsilon}}^{p} \\
& \hat{\boldsymbol{P}}_{2}^{\mathrm{p}}=\int_{\Gamma_{2}} \boldsymbol{\psi}_{u}^{\mathrm{T}} \int_{\Omega} \mathbf{G}_{t \sigma} \overline{\boldsymbol{\varepsilon}}^{p} \\
& \hat{\boldsymbol{W}}_{0}^{\mathrm{p}}=\int_{\Gamma_{0}} \boldsymbol{\psi}_{f}^{\mathrm{T}} \int_{\Omega} \mathbf{G}_{u \sigma} \overline{\boldsymbol{\varepsilon}}^{p} \\
& \hat{\boldsymbol{L}}_{0}^{\mathrm{p}}=\int_{\Gamma_{0}} \boldsymbol{\psi}_{u}^{\mathrm{T}} \int_{\Omega} \mathbf{G}_{t \sigma} \overline{\boldsymbol{\varepsilon}}^{p}
\end{aligned}
$$

The matrix $\mathbf{G}_{\mathrm{hk}}$ collects the fundamental solutions (FSs) in which the effect is specified by the first index $h$, the cause is specified by the dual quantity associated with the second index $k$, where $h, k=u$, $t, \sigma$.

In equation (7), the terms $\overline{\mathbf{A}}_{u 0, f 0}=\overline{\mathbf{A}}_{f 0, u 0}^{\mathrm{T}}$ include the weightings of the CPV integrals and the corresponding free terms.

It is easy to observe from equations (7) to (9) that the coefficients are computed as double integrals. Some of the integrals constituting the matrix $\mathbf{A}$ in equation (6) show singular or hyper-singular kernels and this is a result of the cause distribution acting on 
the same boundary elements as those where the weighted effect is valued.

These difficulties have been removed through a strategy developed by Panzeca et al. [27, 28], subsequently clarified in detail by Terravecchia [29], to obtain all the coefficients of equation (6) in closed form. This strategy lead to the development of a program called Karnak.sGbem [26] that is able to give solutions to plane structural systems possessing any geometry and subjected to boundary and domain actions in the presence of both elastic and inelastic material behaviour.

Equation (6) may be expressed in an extended form as

$$
\begin{aligned}
& 0=\mathbf{A} \boldsymbol{X}+\mathbf{A}_{0}\left(-\boldsymbol{U}_{0}\right)+\hat{\boldsymbol{L}}^{\mathrm{e}}+\hat{\boldsymbol{L}}^{\mathrm{p}} \\
& \boldsymbol{P}_{0}=\tilde{\mathbf{A}}_{0} \boldsymbol{X}+\mathbf{A}_{00}\left(-\boldsymbol{U}_{0}\right)+\hat{\boldsymbol{L}}_{0}^{\mathrm{e}}+\hat{\boldsymbol{L}}_{0}^{\mathrm{p}}
\end{aligned}
$$

where the vector $\boldsymbol{X}$ collects the boundary subvectors $\boldsymbol{F}_{1},\left(-\boldsymbol{U}_{2}\right)$, and $\boldsymbol{F}_{0}$, whereas $\left(-\boldsymbol{U}_{0}\right)$ collects the displacements of the nodes in the interface zone, changed in sign. The vector $\boldsymbol{P}_{0}$ represents the generalized (or weighted) traction vector defined in the boundary elements of the same interface zone, obtained as a response to all the known and unknown actions, with regard to boundary and domain quantities.

Performing a variables condensation through the substitution of the $\boldsymbol{X}$ vector extracted from equation (10a) into equation (10b) leads to

$$
\boldsymbol{P}_{0}=\mathbf{D}_{00} \boldsymbol{U}_{0}+\hat{\boldsymbol{P}}_{0}^{\mathrm{e}}+\hat{\boldsymbol{P}}_{0}^{\mathrm{p}}
$$

where the following conditions are made

$$
\mathbf{D}_{00}=\mathbf{A}_{0}^{\mathrm{T}} \mathbf{A}^{-1} \mathbf{A}_{0}-\mathbf{A}_{00}
$$

$$
\begin{aligned}
& \hat{\boldsymbol{P}}_{0}^{\mathrm{e}}=\hat{\boldsymbol{L}}_{0}^{\mathrm{e}}-\mathbf{A}_{0}^{\mathrm{T}} \mathbf{A}^{-1} \hat{\boldsymbol{L}}^{\mathrm{e}} \\
& \hat{\boldsymbol{P}}_{0}^{\mathrm{p}}=\hat{\boldsymbol{L}}_{0}^{\mathrm{p}}-\mathbf{A}_{0}^{\mathrm{T}} \mathbf{A}^{-1} \hat{\boldsymbol{L}}^{\mathrm{p}}
\end{aligned}
$$

Equation (11) is called the characteristic equation of the bem-e. It relates the generalized (or weighted) tractions $\boldsymbol{P}_{0}$ defined on the boundary elements of the interface zone $\Gamma_{0}$ to the displacements $\boldsymbol{U}_{0}$ of the nodes of the same boundary and to the two load terms $\hat{\boldsymbol{P}}_{0}^{\mathrm{e}}$ and $\hat{\boldsymbol{P}}_{0}^{\mathrm{p}}$ (Fig. 1). These latter terms represent the generalized traction vectors, as elastic and plastic effects, evaluated on the same boundary elements. Moreover, $\mathbf{D}_{00}$ is the stiffness matrix of the examining bem-e.

Equation (11), which is valid for each bem-e, is formally identical to the expression written for the finite elements and it only involves quantities related to the interface zones.

Thus, the proposed approach is able to:

(a) compute all the coefficients of the stiffness matrix and of the loading vectors in equation (11) in closed form;

(b) modify the dimensions of the matrices and vectors involved in the analysis process, since they only depend on the nodal variables in the interface zone.

\subsection{Solution procedure}

Once equation (11) is obtained, the following strategy allows the system to be solved using only the kinematical variables of the interface zones.

The solution procedure begins with a subdivision of the body into $n$ bem-es (Fig. 1) and then equation (11) is rewritten in terms of each of these elements

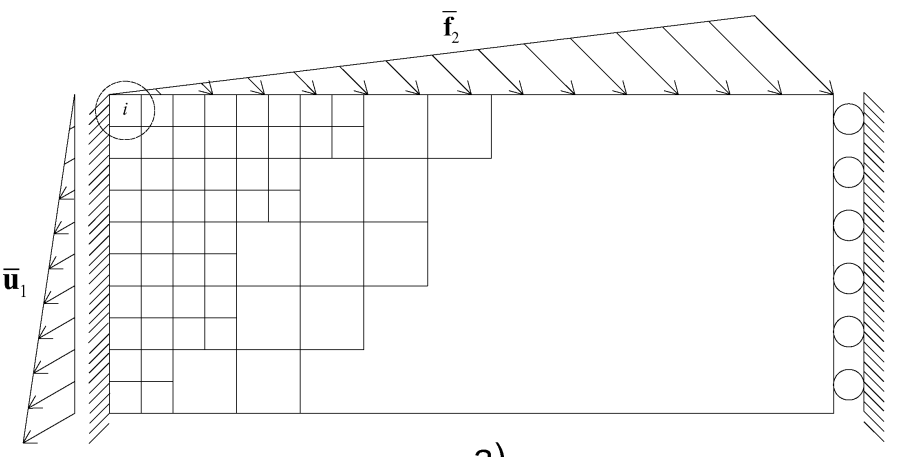

a)

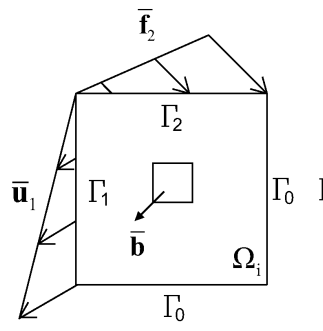

b)

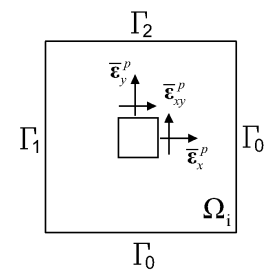

c)

Fig. 1 (a) The body subdivided in bem-es, (b) the $i$ th bem-e subjected to external actions, and (c) the $i$ th bem-e subjected to plastic actions 


$$
\boldsymbol{P}^{i}=\mathbf{D}^{i} \boldsymbol{U}^{i}+\left(\hat{\boldsymbol{P}}^{\mathrm{e}}\right)^{i}+\left(\hat{\boldsymbol{P}}^{\mathrm{p}}\right)^{i} \text { for } i=1, \ldots, n
$$

where the subscripts have been omitted for simplicity.

Thus, a global relationship is obtained that connects all the generalized tractions to all the nodal displacements in the interface zones

$$
\boldsymbol{P}=\mathbf{D} \boldsymbol{U}+\hat{\boldsymbol{P}}^{\mathrm{e}}+\hat{\boldsymbol{P}}^{\mathrm{p}}
$$

where

$$
\begin{aligned}
& \boldsymbol{P}=\left|\begin{array}{c}
\boldsymbol{P}^{1} \\
\vdots \\
\boldsymbol{P}^{n}
\end{array}\right|, \quad \mathbf{D}=\left|\begin{array}{lll}
\mathbf{D}^{1} & & \\
& \ddots & \\
& & \mathbf{D}^{n}
\end{array}\right|, \quad \boldsymbol{U}=\left|\begin{array}{c}
\boldsymbol{U}^{1} \\
\vdots \\
\boldsymbol{U}^{n}
\end{array}\right|, \\
& \hat{\boldsymbol{P}}^{\mathrm{e}}=\left|\begin{array}{c}
\left(\hat{\boldsymbol{P}}^{\mathrm{e}}\right)^{1} \\
\vdots \\
\left(\hat{\boldsymbol{P}}^{\mathrm{e}}\right)^{n}
\end{array}\right|, \quad \hat{\boldsymbol{P}}^{\mathrm{p}}=\left|\begin{array}{c}
\left(\hat{\boldsymbol{P}}^{\mathrm{p}}\right)^{1} \\
\vdots \\
\left(\hat{\boldsymbol{P}}^{\mathrm{p}}\right)^{n}
\end{array}\right|
\end{aligned}
$$

Compatibility among the nodal displacements of adjacent bem-es is introduced using

$$
\boldsymbol{U}=\mathbf{H} \boldsymbol{\xi}
$$

where $\mathbf{H}$ is a topological matrix and $\boldsymbol{\xi}$ the nodal displacements vector of the assembled system.

The equilibrium conditions among generalized forces on the interface boundary may be written in the following form

$$
\mathbf{H}^{\mathrm{T}} \boldsymbol{P}=0
$$

Equations (14), (16), and (17) lead to

$$
\mathbf{K} \boldsymbol{\xi}+\hat{\boldsymbol{f}}^{\mathrm{e}}+\hat{\boldsymbol{f}}^{\mathrm{p}}=0
$$

where $\mathbf{K}=\mathbf{H}^{\mathrm{T}} \mathbf{D} \mathbf{H}$ is the stiffness matrix of the system, and $\hat{\boldsymbol{f}}^{\mathrm{e}}=\mathbf{H}^{\mathrm{T}} \hat{\boldsymbol{p}}^{\mathrm{e}}$ and $\hat{\boldsymbol{f}}^{\mathrm{p}}=\mathbf{H}^{\mathrm{T}} \hat{\boldsymbol{p}}^{\mathrm{p}}$ are the elastic and plastic generalized load vectors, respectively.

The remaining nodal quantities, regarding the reactive forces $\boldsymbol{F}_{1}$ on $\Gamma_{1}$, the displacements $\boldsymbol{U}_{2}$ on $\Gamma_{2}$, and the forces $\boldsymbol{F}_{0}$ on $\Gamma_{0}$ (all variables collected in the vector $\boldsymbol{X}$ ), are obtainable by using equations like equation (10a), written for each bem-e.
Now it is possible to evaluate the elastic predictor $\sigma^{*}$ at the Gauss points of each bem-e by using the following stress SI

$$
\begin{aligned}
\boldsymbol{\sigma}^{*}= & \boldsymbol{\sigma}\left(\boldsymbol{F}_{1},-\boldsymbol{U}_{2}, \boldsymbol{F}_{0},-\boldsymbol{U}_{0}\right)+\boldsymbol{\sigma}\left(\overline{\boldsymbol{F}}_{2},-\overline{\boldsymbol{U}}_{1}, \overline{\boldsymbol{b}}\right) \\
& +\boldsymbol{\sigma}\left(\overline{\boldsymbol{\varepsilon}}^{\mathrm{P}}\right) \text { in } \Omega
\end{aligned}
$$

where $\overline{\boldsymbol{\varepsilon}}^{\mathrm{P}}$ is the plastic strain vector, imposed inside each bem-e and its evaluation will be explained in the following section.

\section{A RETURN MAPPING ALGORITHM FOR ELASTO-PLASTICITY}

The elastic-plastic solution utilizes a strain-driven strategy characterized by two phases:

(a) the evaluation of the elastic predictor using the analysis method discussed in Section 2;

(b) the evaluation of the corrector using a return mapping algorithm.

Since the elastic-plastic response is path-dependent, the solution is difficult to obtain if a space and time discretization is not used.

Assume that the starting conditions are a stress $\sigma_{n}$, total $\varepsilon_{n}$, and plastic $\varepsilon_{n}^{\mathrm{p}}$ strains related at instant $t_{n}$. The unknown response in terms of $\boldsymbol{\sigma}_{n+1}, \varepsilon_{n+1}$, and $\varepsilon_{n+1}^{\mathrm{p}}$ at instant $t_{n+1}$ has to be evaluated. The integration of $\dot{\boldsymbol{\varepsilon}}^{\mathrm{p}}$ in the interval $\left[t_{n}, t_{n+1}\right]$ leads to the following relation

$$
\varepsilon_{n+1}^{\mathrm{p}}=\varepsilon_{n}^{\mathrm{p}}+\int_{t_{n}}^{t_{n+1}} \dot{\gamma} \frac{\partial F}{\partial \boldsymbol{\sigma}} \mathrm{d} t
$$

where $F$ is the yield function and $\dot{\gamma}$ the plastic multiplier increment.

Integrating by parts and assuming the condition $\gamma\left[t_{n}\right]=0$, it follows that

$$
\varepsilon_{n+1}^{\mathrm{p}}=\varepsilon_{n}^{\mathrm{p}}+\left.\gamma_{n+1} \frac{\partial F}{\partial \boldsymbol{\sigma}}\right|_{n+1}-\int_{t_{n}}^{t_{n+1}} \dot{\gamma} \frac{\mathrm{d}}{\mathrm{d} t} \frac{\partial F}{\partial \boldsymbol{\sigma}} \mathrm{d} t
$$

Assume that the loading history is defined by an extremal path [17, 18], as in Ponter and Martin [20]. The approach is based on the exact integration of the constitutive law $\boldsymbol{\sigma}=\mathbf{E}\left(\varepsilon-\varepsilon^{\mathrm{p}}\right)$ either along a particular stress path which maximizes the complementary work or along a particular strain path which minimizes the deformation work, $\mathbf{E}$ being the elasticity operator. Therefore, the extremal paths are elastic ones connecting, in the stress space, the starting point $\sigma_{n}$ and the final point $\sigma_{n+1}$, assuming that the 
plastic process takes place at the final point. Thus, equation (21) takes the form of

$$
\varepsilon_{n+1}^{\mathrm{p}}=\varepsilon_{n}^{\mathrm{p}}+\left.\gamma_{n+1} \frac{\partial F}{\partial \sigma}\right|_{n+1}
$$

As a consequence, under the extremal path hypothesis the plastic response is path-independent.

The elastic predictor $\sigma_{n+1}^{*}$ is a function of the initial conditions $\sigma_{n}$ and the load increment, that is

$$
\boldsymbol{\sigma}_{n+1}^{*}=\sigma_{n}+\mathbf{E} \Delta \varepsilon(\Delta \boldsymbol{u})
$$

If $F\left(\boldsymbol{\sigma}_{n+1}^{*}\right)>0$, the stress has to be modified and a return mapping algorithm is required. In the present approach, such an algorithm utilizes equation (22) and is obtained by solving the following non-linear equation system having the three stress components of $\sigma_{n+1}$ and the plastic multiplier $\gamma_{n+1}$ as unknowns

$$
\left\{\begin{array}{l}
\boldsymbol{\sigma}_{n+1}-\boldsymbol{\sigma}_{n+1}^{*}+\left.\gamma_{n+1} \mathbf{E} \frac{\partial \mathrm{F}}{\partial \boldsymbol{\sigma}}\right|_{n+1}=\boldsymbol{O} \\
F\left(\boldsymbol{\sigma}_{n+1}\right)=0
\end{array}\right.
$$

In particular, with regard to the case of a $2 \mathrm{D}$ continuous solid and under the assumption of a plane strain condition, the elastic-perfectly-plastic von Mises' law is applicable

$$
\begin{gathered}
F\left(\boldsymbol{\sigma}_{n+1}\right)=\frac{1}{2} \boldsymbol{\sigma}_{n+1}^{\mathrm{T}} \mathbf{M} \boldsymbol{\sigma}_{n+1}-\sigma_{y}^{2} \text { with } \\
\mathbf{M}=\left|\begin{array}{ccc}
2 & -1 & 0 \\
-1 & 2 & 0 \\
0 & 0 & 6
\end{array}\right|
\end{gathered}
$$

where $\sigma_{y}$ is the yield stress.

Equation (23) now takes the form of

$$
\left\{\begin{array}{l}
\boldsymbol{f}_{\mathrm{I}} \equiv \boldsymbol{\sigma}_{n+1}-\boldsymbol{\sigma}_{n+1}^{*}+\gamma_{n+1} \mathbf{E} \mathbf{M} \boldsymbol{\sigma}_{n+1}=\mathbf{0} \\
f_{\mathrm{II}} \equiv \frac{1}{2} \boldsymbol{\sigma}_{n+1}^{\mathrm{T}} \mathbf{M} \boldsymbol{\sigma}_{n+1}-\sigma_{y}^{2}=0
\end{array}\right.
$$

The approximate solution $\boldsymbol{x}=\left[\begin{array}{ll}\boldsymbol{\sigma}_{n+1}^{\mathrm{T}} & \gamma_{n+1}\end{array}\right]^{\mathrm{T}}$ of this non-linear problem is obtained by applying the standard Newton-Raphson procedure as follows

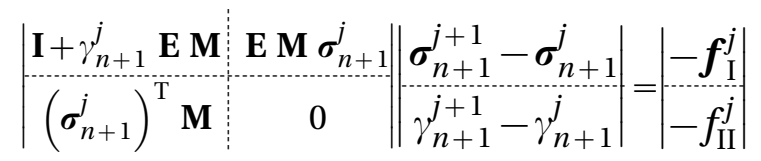

which can be written in the compact form of

$$
\boldsymbol{x}^{j+1}=\boldsymbol{x}^{j}-\mathbf{J}\left(\boldsymbol{x}^{j}\right)^{-1} \boldsymbol{f}\left(\boldsymbol{x}^{j}\right)
$$

where the Jacobian matrix $\mathbf{J}$ contains the derivatives of the functions defined in equation (26), $\boldsymbol{x}^{j+1}$ is the vector of the unknowns, and $\boldsymbol{x}^{j}$ and $\boldsymbol{f}\left(\boldsymbol{x}^{j}\right)$ are the known vectors evaluated at the $j$ th step. Equation (28) allows the evaluation of the plastic strain vector $\bar{\varepsilon}^{\mathrm{P}}$ given in equation (22). Their presence in equations (2) and (3) involves domain integrals that have singular kernels that need to be appropriately handled.

This procedure, derived for the case of plane strain conditions and elastic-perfectly-plastic von Mises' behaviour, has been implemented using the Karnak.sGbem code.

\section{COMPUTATIONAL ASPECTS}

Within the elastoplasticity field treated by the SBEM approach difficulties arise regarding the removal of the strong singularities that occur in the kernels of the domain integrals. They are created when the differential operator, necessary to obtain the stress, is applied to a singular displacement field.

The following new aspects are presented in this paper.

1. Computational aspects regarding the evaluation of the trial stress at the Gauss point, caused by the plastic strain distribution. Indeed, in the SI of the displacements domain integral is regularized and the singular term is transferred to the boundary through the RIM technique. In this way, one obtains a non-singular field of the displacements to which the differential operator may be applied. As a consequence, a non-singular expression for the traction may be obtained in the domain.

2. Computational aspects regarding the weighted traction coefficients present in the symmetric formulation. A new treatment for the evaluation of the traction on the boundary: it is obtained through a limit operation and since the integral has a strong singularity it can be defined as a CPV to which the related Bui free term and an additional free term depending on the boundary geometry are associated.

The mathematical aspects concerning the removal of the strong singularities in the kernels of the double integrals may be found in Panzeca et al. [23].

\subsection{Stress field}

The starting point of the elastic problem is the SI of the displacements 


$$
\begin{aligned}
\boldsymbol{u}\left(\boldsymbol{f},-\boldsymbol{u}, \overline{\boldsymbol{b}}^{,}, \overline{\boldsymbol{\varepsilon}}^{\mathrm{P}}\right)= & \int_{\Gamma} \mathbf{G}_{u u} \boldsymbol{f} \mathrm{d} \Gamma+\int_{\Gamma} \mathbf{G}_{u t}(-\boldsymbol{u}) \mathrm{d} \Gamma \\
& +\int_{\Omega} \mathbf{G}_{u u} \overline{\boldsymbol{b}} \mathrm{d} \Omega+\int_{\Omega} \mathbf{G}_{u \sigma} \overline{\overline{\boldsymbol{\varepsilon}}}^{\mathrm{P}} \mathrm{d} \Omega
\end{aligned}
$$

This equation provides the displacements in $\Omega_{\infty}$ caused not only by the mechanical $\boldsymbol{f}$ and kinematical $-\boldsymbol{u}$ discontinuity vectors, both containing known and unknown quantities, but also by the plastic strains $\bar{\varepsilon}^{\mathrm{p}}$ defined by using the strategy discussed in Section 3. The SIs of the strains, stresses, and tractions can be obtained using equation (29)

The compatibility condition gives the following strain field

$$
\begin{aligned}
\boldsymbol{\varepsilon} & =\varepsilon^{\mathrm{t}}-\overline{\boldsymbol{\varepsilon}}_{Q}^{\mathrm{p}}=\boldsymbol{\varepsilon}^{\mathrm{t}}\left(\boldsymbol{f},-\boldsymbol{u}, \overline{\boldsymbol{b}}, \bar{\varepsilon}^{\mathrm{p}}\right)-\overline{\boldsymbol{\varepsilon}}_{Q}^{\mathrm{p}} \\
& =\mathbf{D}_{\boldsymbol{x}} \boldsymbol{u}\left(\boldsymbol{f},-\boldsymbol{u}, \overline{\boldsymbol{b}}, \bar{\varepsilon}^{\mathrm{p}}\right)-\overline{\boldsymbol{\varepsilon}}_{Q}^{\mathrm{p}}
\end{aligned}
$$

where $\boldsymbol{u}\left[\boldsymbol{f},-\boldsymbol{u}, \overline{\boldsymbol{\varepsilon}}^{\mathrm{p}}\right]$ is defined by equation (29) and $\overline{\boldsymbol{\varepsilon}}_{\mathrm{Q}}^{\mathrm{p}}$ is the known plastic action at point $Q$. The differential operator $\mathbf{D}_{x}$ provides the following integral equation

$$
\begin{aligned}
\boldsymbol{\varepsilon}= & \int_{\Gamma} \mathbf{G}_{\varepsilon u} \boldsymbol{f} \mathrm{d} \Gamma+\int_{\Gamma} \mathbf{G}_{\varepsilon t}(-\boldsymbol{u}) \mathrm{d} \Gamma \\
& +\int_{\Omega} \mathbf{G}_{\varepsilon u} \overline{\boldsymbol{b}} \mathrm{d} \Omega+\mathbf{D}_{x} \int_{\Omega} \mathbf{G}_{u \sigma} \overline{\boldsymbol{\varepsilon}}^{\mathrm{p}} \mathrm{d} \Omega-\overline{\boldsymbol{\varepsilon}}_{Q}^{\mathrm{p}}
\end{aligned}
$$

where $\mathbf{G}_{\varepsilon \mathrm{u}}=\mathbf{D}_{\boldsymbol{x}} \mathbf{G}_{\mathrm{uu}}$ and $\mathbf{G}_{\varepsilon \mathrm{t}}=\mathbf{D}_{\boldsymbol{x}} \mathbf{G}_{\mathrm{ut}}$ have been assumed.

By applying the elasticity operator $\mathbf{E}$ and the director cosines matrix $\mathbf{N}$ to equation (31), the stress and traction expressions can be obtained as

$$
\begin{aligned}
\boldsymbol{\sigma} & =\mathbf{E} \boldsymbol{\varepsilon}=\mathbf{E}\left[\mathbf{D}_{\boldsymbol{x}} \boldsymbol{u}\left(\boldsymbol{f},-\boldsymbol{u}, \overline{\boldsymbol{b}}, \overline{\boldsymbol{\varepsilon}}^{\mathrm{p}}\right)-\overline{\boldsymbol{\varepsilon}}_{Q}^{\mathrm{p}}\right] \\
& =\boldsymbol{\sigma}^{\mathrm{t}}\left(\boldsymbol{f},-\boldsymbol{u}, \overline{\boldsymbol{b}}, \overline{\boldsymbol{\varepsilon}}^{\mathrm{p}}\right)-\mathbf{E} \overline{\boldsymbol{\varepsilon}}_{Q}^{\mathrm{p}} \\
\boldsymbol{t} & =\mathbf{N}^{T} \boldsymbol{\sigma}=\mathbf{N}^{T}\left[\boldsymbol{\sigma}^{\mathrm{t}}\left(\boldsymbol{f},-\boldsymbol{u}, \overline{\boldsymbol{b}}, \overline{\boldsymbol{\varepsilon}}^{\mathrm{p}}\right)-\mathbf{E} \overline{\boldsymbol{\varepsilon}}_{Q}^{\mathrm{p}}\right]
\end{aligned}
$$

In the domain integral for the evaluation of the strain $\varepsilon$, caused by the plastic strain field $\bar{\varepsilon}^{\mathrm{p}}$, the differential operator involves the presence of a hyper-singularity through the displacement gradient. If the domain is divided into two sub-domains where $\Omega_{\varepsilon}$ is a circular domain that excludes point $Q$, the last integral in equation (31) can be written as

$$
\begin{aligned}
& \mathbf{D}_{x} \int_{\Omega} \mathbf{G}_{u \sigma} \bar{\varepsilon}^{\mathrm{p}} \mathrm{d} \Omega=\lim _{\varepsilon \rightarrow 0}\left(\mathbf{D}_{x} \int_{\Omega-\Omega_{\varepsilon}} \mathbf{G}_{u \sigma} \bar{\varepsilon}^{\mathrm{p}} \mathrm{d} \Omega\right) \\
& +\lim _{\varepsilon \rightarrow 0}\left(\mathbf{D}_{x} \int_{\Omega_{\varepsilon}} \mathbf{G}_{u \sigma} \mathrm{d} \Omega \cdot \bar{\varepsilon}_{Q}^{\mathrm{p}}\right)
\end{aligned}
$$

where the second integral, transformed into polar coordinates, is equal to zero, the kernel being a circular function.

The remaining integral may be regularized in the following way

$$
\begin{aligned}
\mathbf{D}_{x} \int_{\Omega} \mathbf{G}_{u \sigma} \bar{\varepsilon}^{\mathrm{p}} \mathrm{d} \Omega= & \lim _{\varepsilon \rightarrow 0} \mathbf{D}_{\boldsymbol{x}} \int_{\Omega-\Omega_{\varepsilon}} \mathbf{G}_{u \sigma}\left(\overline{\boldsymbol{\varepsilon}}^{\mathrm{p}}-\overline{\boldsymbol{\varepsilon}}_{Q}^{\mathrm{p}}\right) \mathrm{d} \Omega \\
& +\lim _{\varepsilon \rightarrow 0} \mathbf{D}_{\boldsymbol{x}} \int_{\Omega-\Omega_{\varepsilon}} \mathbf{G}_{u \sigma} \mathrm{d} \Omega \cdot \bar{\varepsilon}_{Q}^{\mathrm{p}}
\end{aligned}
$$

The last integral is transformed into polar coordinates $[22,30,31]$, which results in a double integral; the inner integral is radial and is evaluated analytically and the outer integral is defined through boundary variables. That is to say

$$
\begin{aligned}
\mathbf{D}_{\boldsymbol{x}} \int_{\Omega} \mathbf{G}_{u \sigma} \overline{\boldsymbol{\varepsilon}}^{\mathrm{p}} \mathrm{d} \Omega= & \lim _{\varepsilon \rightarrow 0} \mathbf{D}_{\boldsymbol{x}} \int_{\Omega-\Omega_{\varepsilon}} \mathbf{G}_{u \sigma}\left(\overline{\boldsymbol{\varepsilon}}^{\mathrm{p}}-\overline{\boldsymbol{\varepsilon}}_{Q}^{\mathrm{p}}\right) \mathrm{d} \Omega \\
& +\lim _{\varepsilon \rightarrow 0} \mathbf{D}_{\boldsymbol{x}} \int_{0}^{2 \pi} \int_{0}^{R} \frac{\boldsymbol{\Psi}_{u \sigma}(\varphi)}{r} r \mathrm{~d} r \mathrm{~d} \varphi \cdot \overline{\boldsymbol{\varepsilon}}_{Q}^{\mathrm{p}} \\
= & \lim _{\varepsilon \rightarrow 0} \mathbf{D}_{\boldsymbol{x}} \int_{\Omega-\Omega_{\varepsilon}} \mathbf{G}_{u \sigma}\left(\overline{\boldsymbol{\varepsilon}}^{\mathrm{p}}-\overline{\boldsymbol{\varepsilon}}_{Q}^{\mathrm{p}}\right) \mathrm{d} \Omega \\
& +\lim _{\varepsilon \rightarrow 0} \mathbf{D}_{\boldsymbol{x}} \int_{0}^{2 \pi} \frac{\boldsymbol{\Psi}_{u \sigma}(\varphi)}{R} R^{2} \mathrm{~d} \varphi \cdot \overline{\boldsymbol{\varepsilon}}_{Q}^{\mathrm{p}} \\
= & \int_{\Omega} \mathbf{G}_{\varepsilon \sigma}\left(\overline{\boldsymbol{\varepsilon}}^{\mathrm{p}}-\overline{\boldsymbol{\varepsilon}}_{Q}^{\mathrm{p}}\right) \mathrm{d} \Omega \\
& +\mathbf{D}_{\boldsymbol{x}} \int_{\Gamma} \mathbf{G}_{u \sigma} \boldsymbol{n}^{\mathrm{T}} \boldsymbol{r} \mathrm{d} \Gamma \cdot \overline{\boldsymbol{\varepsilon}}_{Q}^{\mathrm{p}}
\end{aligned}
$$


where the condition $R^{2} \mathrm{~d} \varphi=\boldsymbol{n}^{\mathrm{T}} \boldsymbol{r} \mathrm{d} \Gamma$ has been adopted. A regular field of displacements on which the differential operator may be applied is now required.

In equation (36), the integral

$$
\int_{\Gamma} \mathbf{G}_{u \sigma} \boldsymbol{n}^{\mathrm{T}} \boldsymbol{r} \mathrm{d} \Gamma \cdot \bar{\varepsilon}_{Q}^{\mathrm{p}}
$$

defines a displacement field, whose characteristics are:

1. It is non-singular in $\Omega / \Gamma$, because the integral is evaluated on the boundary, therefore $\boldsymbol{x} \neq \boldsymbol{x}^{\prime}$ is always valid.

2. It is non-singular on $\Gamma$, because when $\left(\boldsymbol{x}, \boldsymbol{x}^{\prime}\right) \in \Gamma$ and $\boldsymbol{x} \rightarrow \boldsymbol{x}^{\prime}$ one has $\boldsymbol{n}^{\mathrm{T}} \boldsymbol{r}=0$.

3. It is continued on $\Gamma$, but the first derivative is discontinuous.

Assuming the condition that $\mathbf{D}_{\boldsymbol{x}}()=.-\mathbf{D}_{\boldsymbol{x}^{\prime}}($.$) , the$ integration by parts of equation (37) results in

$$
\begin{aligned}
\mathbf{D}_{\boldsymbol{x}} \int_{\Gamma} \mathbf{G}_{u \sigma} \boldsymbol{n}^{\mathrm{T}} \boldsymbol{r} \mathrm{d} \Gamma \times \overline{\boldsymbol{\varepsilon}}_{Q}^{\mathrm{p}}= & \int_{\Gamma} \mathbf{G}_{\varepsilon \sigma} \boldsymbol{n}^{\mathrm{T}} \boldsymbol{r} \mathrm{d} \Gamma \cdot \overline{\boldsymbol{\varepsilon}}_{Q}^{\mathrm{p}} \\
& -\int_{\Gamma} \mathbf{N}^{\prime} \mathbf{G}_{u \sigma} \mathrm{d} \Gamma \cdot \bar{\varepsilon}_{Q}^{\mathrm{p}}
\end{aligned}
$$

In this equation, the first integral on the right-hand side is equal to zero because the kernel is circular, that is

$$
\int_{\Gamma} \mathbf{G}_{\varepsilon \sigma} \boldsymbol{n}^{\mathrm{T}} \boldsymbol{r} \mathrm{d} \Gamma=0
$$

The following expressions of the regularized strains and stresses fields can now be obtained

$$
\begin{aligned}
& \boldsymbol{\varepsilon}= \int_{\Gamma} \mathbf{G}_{\varepsilon u} \boldsymbol{f} \mathrm{d} \Gamma+\int_{\Gamma} \mathbf{G}_{\varepsilon t}(-\boldsymbol{u}) \mathrm{d} \Gamma+\int_{\Omega} \mathbf{G}_{\varepsilon u} \overline{\boldsymbol{b}} \mathrm{d} \Omega \\
&+\int_{\Omega} \mathbf{G}_{\varepsilon \sigma}\left(\overline{\boldsymbol{\varepsilon}}^{\mathrm{p}}-\overline{\boldsymbol{\varepsilon}}_{Q}^{\mathrm{p}}\right) \mathrm{d} \Omega-\left(\int_{\Gamma} \mathbf{N}^{\prime} \mathbf{G}_{u \sigma} \mathrm{d} \Gamma+\mathbf{I}\right) \cdot \overline{\boldsymbol{\varepsilon}}_{Q}^{\mathrm{p}} \\
& \boldsymbol{\sigma}=\mathbf{E} \boldsymbol{\varepsilon}=\int_{\Gamma} \mathbf{G}_{\sigma u} \boldsymbol{f} \mathrm{d} \Gamma+\int_{\Gamma} \mathbf{G}_{\sigma t}(-\boldsymbol{u}) \mathrm{d} \Gamma+\int_{\Omega} \mathbf{G}_{\sigma u} \overline{\boldsymbol{b}} \mathrm{d} \Omega \\
&+\int_{\Omega} \mathbf{G}_{\sigma \sigma}\left(\overline{\boldsymbol{\varepsilon}}^{\mathrm{p}}-\bar{\varepsilon}_{Q}^{\mathrm{p}}\right) \mathrm{d} \Omega-\mathbf{E}\left(\int_{\Gamma} \mathbf{N}^{\prime} \mathbf{G}_{u \sigma} \mathrm{d} \Gamma+\mathbf{I}\right) \cdot \bar{\varepsilon}_{Q}^{\mathrm{p}}
\end{aligned}
$$

The SI of the traction on an element defined by $\boldsymbol{n}$ is obtained at an inner point by applying the Cauchy formula to equation (40b)

$$
\begin{aligned}
\boldsymbol{t}= & \int_{\Gamma} \mathbf{G}_{t u} \boldsymbol{f} \mathrm{d} \Gamma+\int_{\Gamma} \mathbf{G}_{t t}(-\boldsymbol{u}) \mathrm{d} \Gamma+\int_{\Omega} \mathbf{G}_{t u} \overline{\boldsymbol{b}} \mathrm{d} \Omega \\
& +\int_{\Omega} \mathbf{G}_{t t}\left(\overline{\boldsymbol{\varepsilon}}^{\mathrm{p}}-\overline{\boldsymbol{\varepsilon}}_{Q}^{\mathrm{p}}\right) \mathrm{d} \Omega-\mathbf{N}^{\mathrm{T}} \mathbf{E}\left(\int_{\Gamma} \mathbf{N}^{\prime} \mathbf{G}_{u \sigma} \mathrm{d} \Gamma+\mathbf{I}\right) \cdot \overline{\boldsymbol{\varepsilon}}_{Q}^{\mathrm{p}}
\end{aligned}
$$

In order to permit an analysis via SBEM, the traction has to be computed on the boundary. The related computational aspects of performing this calculation will be discussed in the following section.

\subsection{SI of the traction on the boundary}

The objective is to evaluate the traction at $\boldsymbol{x} \in \Omega$ on an element defined by a unit normal vector $\boldsymbol{n}$ and caused by the inelastic action $\bar{\varepsilon}^{\mathrm{p}}$ in $\Omega$, using the following equation

$$
\boldsymbol{t}\left(\overline{\boldsymbol{\varepsilon}}^{\mathrm{p}}\right)=-\mathbf{N}^{\mathrm{T}} \mathbf{E} \int_{\Gamma} \mathbf{N}^{\prime} \mathbf{G}_{u \sigma} \mathrm{d} \Gamma \cdot \bar{\varepsilon}_{Q}^{\mathrm{p}}
$$

In order to determine the traction on the boundary, the integral (42) has to be evaluated through a limit operation when the effect point acts on the boundary from the inside, i.e. $(\boldsymbol{x}, \boldsymbol{n}) \rightarrow\left(\boldsymbol{x}^{\prime}, \boldsymbol{n}^{\prime}\right) \in \Gamma$. An analogous strategy to the one in section 4.1 is employed. In order to evaluate the traction on the side $\Gamma_{e i}$ using equation (42), it is necessary to distinguish the sides where the integrations have to be performed: in Fig. 2(a) on the boundary $\Gamma_{e i}$; in Fig. 2(b) on all the remaining boundaries, indicated by $\Gamma_{e j}$, for $j=1, . ., i-1, i+1, . . n$.

Consider the first contribution where the cause (i.e. plastic action), as the quantity transferred from the domain, is distributed on the boundary $\Gamma_{e i}$. Consider the boundary element $\Gamma_{e i}$ defined by $\boldsymbol{n}^{\prime}$, and a point $Q$ in the domain, inside an infinitesimal circle (Fig. 2(a)). An infinitesimal element with a slope defined by $\boldsymbol{n} / / \boldsymbol{n}^{\prime}$ is considered and let $d$ be the distance between the point $Q$ and the side $\Gamma_{e i}$ and $\Gamma_{\varepsilon i}$ be the boundary portion of the circle that has the width $\alpha_{1}+\alpha_{2}=2 \pi-\alpha$.

The limit operation of the integral (42) leads to 

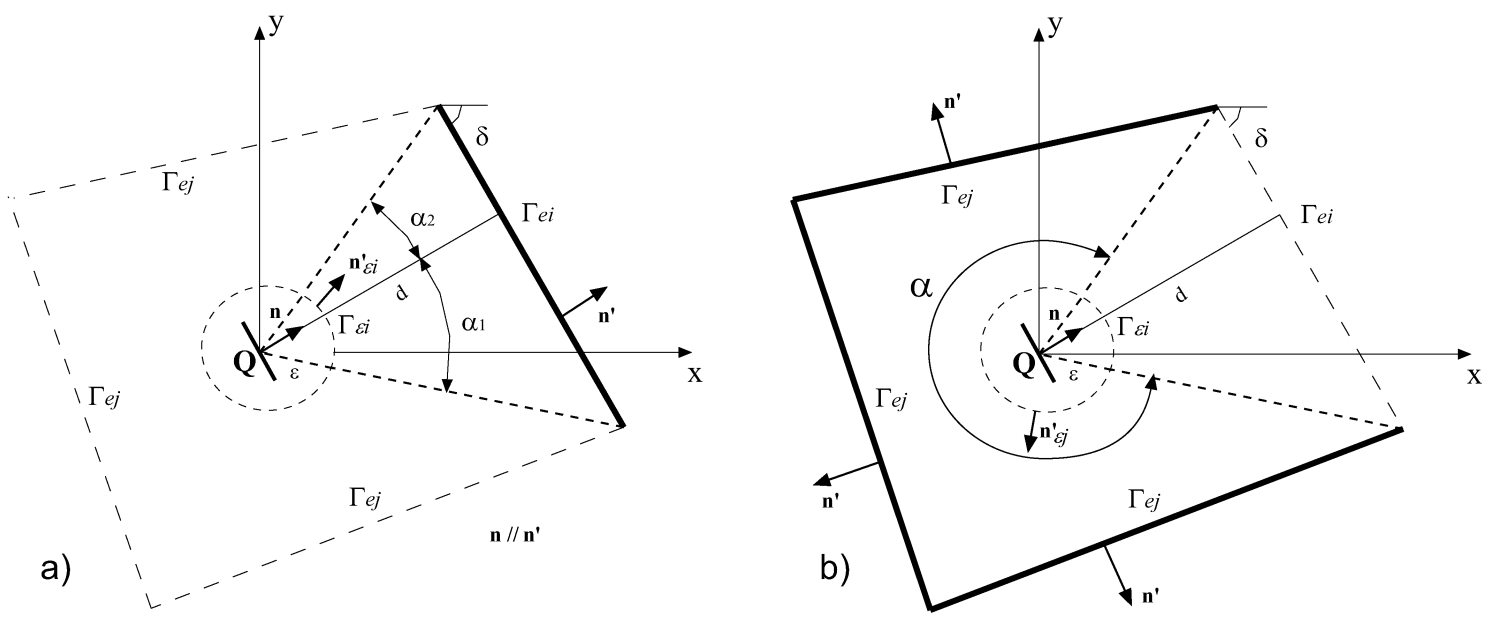

Fig. 2 Traction on the boundary, sum of two integrations: (a) on the boundary where the traction has to be evaluated and (b) on the remaining sides

$$
\begin{aligned}
\boldsymbol{t}\left(\overline{\boldsymbol{\varepsilon}}^{\mathrm{p}}\right) & =-\mathbf{N}^{\mathrm{T}} \mathbf{E} \lim _{d \rightarrow 0^{-}} \int_{\Gamma_{e i}} \mathbf{N}^{\prime} \mathbf{G}_{u \sigma} \mathrm{d} \Gamma_{i} \\
& =-\mathbf{N}^{\mathrm{T}} \mathbf{E} \oint_{\Gamma_{e i}} \mathbf{N}^{\prime} \mathbf{G}_{u \sigma} \mathrm{d} \Gamma_{i}-\mathbf{N}^{\mathrm{T}} \mathbf{E} \mathbf{J}_{\mathrm{b}}+\frac{1}{2} \mathbf{N}^{\mathrm{T}} \mathbf{E} \mathbf{J}
\end{aligned}
$$

where the matrices $\mathbf{J}$ and $\mathbf{J}_{\mathrm{b}}$ are the Bui free term matrix and the slope matrix, respectively

$$
\begin{gathered}
\mathbf{J}=\frac{1}{4(1-v)}\left|\begin{array}{ccc}
\frac{5-4 v}{2} & \frac{-1+4 v}{2} & 0 \\
\frac{1+4 v}{2} & \frac{5-4 v}{2} & 0 \\
0 & 0 & 3-4 v
\end{array}\right| \\
\mathbf{J}_{\mathrm{b}}=\frac{1}{4(1-v)}\left[\begin{array}{c}
-(1-v) \cos (2 \delta)+\frac{1}{4} \cos (4 \delta) \\
-v \cos (\delta)-\frac{1}{4} \cos (4 \delta) \\
-4 \cos ^{3}(\delta) \sin (\delta)
\end{array}\right.
\end{gathered}
$$

$$
\begin{aligned}
- & \mathbf{N}^{\mathrm{T}} \mathbf{E}\left\{\lim _{\mathrm{d} \rightarrow 0^{-}}\left[\lim _{\varepsilon \rightarrow 0} \int_{\alpha i} \mathbf{N}_{\varepsilon i}^{\prime}(\varphi) \frac{\boldsymbol{\Psi}_{u \sigma}}{\not \varnothing} \not \dot{\phi} \mathrm{d} \varphi\right]\right\} \\
= & -\mathbf{N}^{\mathrm{T}} \mathbf{E}\left\{\lim _{d \rightarrow 0^{-}}\left[\int_{\alpha_{i}} \mathbf{N}_{\varepsilon i}^{\prime}(\varphi) \boldsymbol{\Psi}_{u \sigma}(\varphi) \mathrm{d} \varphi\right]\right\} \\
& -\mathbf{N}^{\mathrm{T}} \mathbf{E}\left\{\lim _{\mathrm{d} \rightarrow 0^{-}}\left[\int_{-\left(\alpha_{1}-[(\pi / 2)-\delta)\right)}^{\alpha_{2}+[(\pi / 2)-\delta]} \mathbf{N}_{\varepsilon i}^{\prime}(\varphi) \boldsymbol{\Psi}_{u \sigma}(\varphi) \mathrm{d} \varphi\right]\right\} \\
= & -\mathbf{N}^{\mathrm{T}} \mathbf{E}\left[\int_{-\delta}^{\pi-\delta} \mathbf{N}_{\varepsilon i}^{\prime}(\varphi) \boldsymbol{\Psi}_{u \sigma}(\varphi) \mathrm{d} \varphi\right]=\frac{1}{2} \mathbf{N}^{\mathrm{T}} \mathbf{E} \mathbf{J}
\end{aligned}
$$

where, for $d \rightarrow 0$, the angles are $\alpha_{1}=\alpha_{2}=\pi / 2$.

Now consider the second contribution where the

$$
\left.\begin{array}{cc}
+v \cos (\delta)-\frac{1}{4} \cos (4 \delta) & -\frac{1}{2}[1-2 v+\cos (2 \delta)] \sin (2 \delta) \\
-(1-v) \cos (2 \delta)+\frac{1}{4} \cos (4 \delta) & -\frac{1}{2}[1-2 v-\cos (2 \delta)] \sin (2 \delta) \\
-4 \cos (\delta) \sin ^{3}(\delta) & -\frac{1}{2} \cos (4 \delta)
\end{array}\right]
$$

The term $\mathbf{N}^{\mathrm{T}} \mathbf{E}\left(-\mathbf{J}_{\mathrm{b}}+\mathbf{J} / 2\right)$ is the free term associated with the CPV and depends on:

(a) $-\mathbf{N}^{\mathrm{T}} \mathbf{E} \mathbf{J}_{\mathrm{b}}$ containing trigonometric functions connected to the slope of the boundary element on which the traction has to be evaluated;

(b) the Bui free term $\mathbf{N}^{\mathrm{T}} \mathbf{E} \mathbf{J} / 2$ which is the contribution of the cause distribution related to the side on which the effect point is set.

With reference to Fig. 2(a), the contribution of $\mathbf{N}^{\mathrm{T}} \mathbf{E} \mathbf{J} / 2$ can be obtained as follows cause is distributed on all the remaining boundaries, indicated by $\Gamma_{e j}$. Since in the limit operation the angle between the sides on which the integration is made tends to $\pi$ by referring to Fig. 2(b) the contribution of the other sides can be written as

$$
\begin{aligned}
& -\mathbf{N}^{\mathrm{T}} \mathbf{E}\left\{\sum_{i=2}^{n} \lim _{\mathrm{d} \rightarrow 0^{-}}\left[\int_{\alpha_{i}} \mathbf{N}_{\varepsilon i}^{\prime}(\varphi) \frac{\boldsymbol{\Psi}_{u \sigma}(\varphi)}{\not \varnothing} \not \mathrm{d} \varphi\right]\right\} \\
& =-\mathbf{N}^{\mathrm{T}} \mathbf{E}\left[\int_{\pi-\delta}^{2 \pi-\delta} \mathbf{N}^{\prime}(\varphi) \boldsymbol{\Psi}_{u \sigma}(\varphi) \mathrm{d} \varphi\right]=\frac{1}{2} \mathbf{N}^{\mathrm{T}} \mathbf{E} \mathbf{J}
\end{aligned}
$$


Therefore, the two contributions result in the following equation

$$
\boldsymbol{t}\left(\overline{\boldsymbol{\varepsilon}}^{\mathrm{p}}\right)=-\mathbf{N}^{\mathrm{T}} \mathbf{E} \oint_{\Gamma_{e i}} \mathbf{N}^{\prime} \mathbf{G}_{u \sigma} \mathrm{d} \Gamma_{i}+\mathbf{N}^{\mathrm{T}} \mathbf{E}\left(-\mathbf{J}_{\mathbf{b}}+\mathbf{J}\right)
$$

It can be observed that when the traction is computed at a point $Q$ on a boundary, the contribution to the Bui free term is divided into two equal parts: the first part arises when the cause integral is computed on the same side, the second when the cause integrals reflect the remaining sides. Their values are equal.

Under the assumption that $\bar{\varepsilon}^{\mathrm{p}}=$ const., the SIs may be rewritten to have the form

$$
\begin{aligned}
& \boldsymbol{u}^{\mathrm{e}}(\boldsymbol{f},-\boldsymbol{u}, \overline{\boldsymbol{b}})+\boldsymbol{u}^{\mathrm{p}}\left(\overline{\boldsymbol{\varepsilon}}^{\mathrm{p}}\right)= \int_{\Gamma} \mathbf{G}_{u u} \boldsymbol{f} \mathrm{d} \Gamma+\oint_{\Gamma} \mathbf{G}_{u t}(-\boldsymbol{u}) \\
&+\frac{1}{2} \boldsymbol{u}+\int_{\Gamma} \tilde{\mathbf{G}}_{u u} \overline{\boldsymbol{b}} \mathrm{d} \Gamma \\
&+\int_{\Gamma} \mathbf{G}_{u \sigma}\left(\boldsymbol{n}^{\mathrm{T}} \boldsymbol{r}\right) \mathrm{d} \Gamma \cdot \overline{\boldsymbol{\varepsilon}}_{Q}^{\mathrm{p}} \\
& \boldsymbol{t}^{\mathrm{e}}(\boldsymbol{f},-\boldsymbol{u}, \overline{\boldsymbol{b}})+\boldsymbol{t}^{\mathrm{p}}\left(\overline{\bar{\varepsilon}}^{\mathrm{p}}\right) \\
&=\oint_{\Gamma} \mathbf{G}_{t u} \boldsymbol{f} \mathrm{d} \Gamma+\frac{1}{2} \boldsymbol{f}+\int_{\Gamma} \mathbf{G}_{t t}(-\boldsymbol{u}) \mathrm{d} \Gamma \\
& \quad+\int_{\Gamma} \tilde{\mathbf{G}}_{t u} \overline{\boldsymbol{b}} \mathrm{d} \Gamma-\mathbf{N}^{\mathrm{T}} \mathbf{E} \oint_{\Gamma} \mathbf{N}^{\prime} \mathbf{G}_{u \sigma} \mathrm{d} \Gamma \cdot \bar{\varepsilon}_{Q}^{\mathrm{p}} \\
& \quad+\mathbf{N}^{\mathrm{T}} \mathbf{E}\left(-\mathbf{J}_{\mathrm{b}}+\mathbf{J}-\mathbf{I}\right) \cdot \bar{\varepsilon}_{Q}^{\mathrm{p}}
\end{aligned}
$$

where the vectors $\boldsymbol{f}$ and $-\boldsymbol{u}$ are known and unknown quantities related to the boundaries $\Gamma_{1}, \Gamma_{2}$, and $\Gamma_{0}$.

In equations (48a) and (48b), the body force is transferred onto the boundary and this is discussed in more detail in the Appendix.

Using a symmetric Galerkin BEM, the weightings of $\boldsymbol{u}^{\mathrm{e}}$ and $\boldsymbol{t}^{\mathrm{e}}$ are the coefficients in equation (7) and equations (8a) to (8d), respectively, whereas the weightings of $\boldsymbol{u}^{\mathrm{p}}$ and $\boldsymbol{t}^{\mathrm{p}}$ are the coefficients obtained using equations (9a) to (9d).

\section{THE ELASTOPLASTIC PROCEDURE}

In this section, the sequence of steps that must be performed to obtain the numerical results, is described.
Step 0: Load update

$$
\beta_{n+1}=\beta_{n}+\Delta \beta ; \quad \beta_{n+1} \hat{\boldsymbol{f}}^{\mathrm{e}} ; \quad \beta_{n+1} \hat{\boldsymbol{L}}^{e}
$$

Step 1: Elastic analysis (pre-processing)

$$
\left\{\begin{array}{l}
\boldsymbol{\xi}_{n+1}=-\mathbf{K}^{-1}\left(\beta_{n+1} \hat{\boldsymbol{f}}^{\mathrm{e}}+\hat{\boldsymbol{f}}_{n+1}^{\mathrm{p}(i)}\right) \\
\boldsymbol{X}_{n+1}=-\mathbf{A}^{-1}\left[\mathbf{A}_{0}\left(-\boldsymbol{U}_{n+1}\right)+\beta_{n+1} \hat{\boldsymbol{L}}^{\mathrm{e}}+\hat{\boldsymbol{L}}_{n+1}^{\mathrm{p}(i)}\right]
\end{array}\right.
$$

with

$$
\boldsymbol{U}_{n+1}=\mathbf{H} \xi_{n+1}
$$

(see equations (10) and (18)).

Step 2: Compute the elastic predictor (post-processing)

$$
\boldsymbol{\sigma}_{n+1}^{*}=\boldsymbol{\sigma}(-\boldsymbol{\xi}, \boldsymbol{X})_{n+1}^{(i)}+\beta_{n+1} \boldsymbol{\sigma}\left(\overline{\boldsymbol{F}}_{2},-\overline{\boldsymbol{U}}_{1}, \overline{\boldsymbol{b}},\right)+\boldsymbol{\sigma}\left(\overline{\boldsymbol{\varepsilon}}^{\mathrm{P}(i)}\right)_{n+1}
$$

(see equation (40b)).

Step 3: Check for yielding

If $F\left(\sigma_{n+1}^{*}\right) \leqslant T o l$, go to next load update or EXIT

If $F\left(\boldsymbol{\sigma}_{n+1}^{*}\right)>T o l$, go to step 4

Step 4: Plastic corrector

$$
\boldsymbol{x}^{j+1}=\boldsymbol{x}^{j}-\mathbf{J}\left(\boldsymbol{x}^{j}\right)^{-1} \boldsymbol{f}\left(\boldsymbol{x}^{j}\right) \text { for } \mathbf{j}=1,2, \ldots, \text { Tol }
$$

$$
\Delta \varepsilon_{n+1}^{\mathrm{p}(i)}=\left.\gamma_{n+1}^{(i)} \frac{\partial F}{\partial \boldsymbol{\sigma}^{(i)}}\right|_{n+1}
$$

(see equations (22) and (28)).

Step 5: Plastic strain update

$$
\overline{\boldsymbol{\varepsilon}}_{n+1}^{\mathrm{p}^{(i+1)}}=\overline{\boldsymbol{\varepsilon}}_{n+1}^{\mathrm{p}(i)}+\Delta \overline{\boldsymbol{\varepsilon}}_{n+1}^{\mathrm{p}(i)}
$$

Step 6: Plastic generalized load vectors update

$$
\hat{\boldsymbol{f}}_{n+1}^{\mathrm{p}}\left[\overline{\boldsymbol{\varepsilon}}_{n+1}^{\mathrm{p}(i+1)}\right] ; \quad \hat{\boldsymbol{L}}_{n+1}^{\mathrm{p}}\left[\overline{\boldsymbol{\varepsilon}}_{n+1}^{\mathrm{p}(i+1)}\right]
$$

go to step 1. 


\section{NUMERICAL RESULTS}

In order to show the efficiency of the proposed method, three numerical tests have been performed under the following hypotheses: plane strain condition, elastic-perfectly-plastic von Mises' law, and associated plastic flow.

These applications highlight the performance of the eight-node bem-e that is used in the zones where the plasticity is active. Its use allows a perfectly plastic behaviour of the body to be obtained, i.e. it is coherent with the adopted constitutive law, in a limited number of iterations.

The iterative elasto-plastic analysis carried out via SBEM has a higher computational cost than those for commercial programs that utilize the FEM, however, it does have a higher accuracy for the response. This is a result of using FSs which guarantee the equilibrium and compatibility conditions at inner points of each bem-e. Another advantage of the present approach is that discretization is only used in those parts of the domain where the plastic phenomenon takes place leaving the remaining parts as macro-elements. This approach allows the elastic behaviour to be explained using a reduced number of boundary variables.

\subsection{Plate with circular hole}

In this example, a square plate with a circular hole is subjected to a tensile load $q=100 \mathrm{MPa}$, as shown in Fig. 3. The material characteristics are a Young's modulus $E=206000 \mathrm{MPa}$, a Poisson's ratio $v=0.29$, and a uniaxial yield value $\sigma_{\mathrm{y}}=450 \mathrm{MPa}$. The plate geometry, shown in Fig. 3(a), has unit thickness. Three different types of meshes were considered:

(a) the first mesh had 48 bem-es, see Fig. 3(b);

(b) the second mesh had 80 bem-es, see Fig. 3(c);

(c) the third mesh had 144 bem-es, see Fig. 3(d).

Two different elasto-plastic analyses were performed: first, with a four-node bem-e and a maximum of 300 iterations, and second with an eightnode bem-e and 30 iterations.

The calculated load-displacement curves are shown in Fig. 4. The use of the four-node bem-e leads to very poor results in that it does not create a good elasto-plastic response, which is due its stiffer behaviour than for the eight-node case. The eightnode case resulted in an elastic-perfectly-plastic response of the structure, i.e. in a coherent manner with the adopted constitutive law.

The following points are apparent from studying Fig. 4.
1. In order to fully characterize the load growth it should be incremented in steps of 0.1 , for the eight-node bem-e system the growth curve is almost a horizontal straight line.

2. The progressive refinement of the mesh (i.e. going from 48 bem-es to 144 bem-es) involves decreasing the load multiplier value, which gives rise to the start of the plastic phase.

The stress-total strain curves are plotted in Fig. 5, and the stress and the plastic strain located at the corner of the hole using the third mesh (Fig. 3(d)) as a function of number of iterations and load increment is plotted in Fig. 6 .

From these figures, it is possible to draw the following conclusions.

1. The constitutive behaviours $\sigma_{x}=\sigma_{x}\left(\varepsilon_{x}^{\mathrm{t}}\right), \quad \sigma_{y}=$ $\sigma_{y}\left(\varepsilon_{y}^{\mathrm{t}}\right)$, and $\boldsymbol{\sigma}_{\mathrm{xy}}=\sigma_{\mathrm{xy}}\left(\varepsilon_{\mathrm{xy}}^{\mathrm{t}}\right)$ of Fig. 5 are the sum of the elastic and plastic parts $\varepsilon_{i}^{\mathrm{t}}=\varepsilon_{i}^{\mathrm{e}}+\varepsilon_{i}^{\mathrm{p}}$, where $i=x, y, x y$. The stress in the $y$-direction, obtained through the return mapping strategy proposed in this paper, has an almost constant value $\sigma_{y}=$ 495.3 MPa with a total strain $\varepsilon_{y}^{\mathrm{t}}=0.002584$. The stresses $\boldsymbol{\sigma}_{y}$ and $\boldsymbol{\sigma}_{x y}$ show non-linear behaviour for very low strain values.

2. The variations in the stress $\boldsymbol{\sigma}_{y}=\boldsymbol{\sigma}_{y}(\beta)$ and the plastic strain $\varepsilon_{y}^{\mathrm{p}}=\varepsilon_{y}^{\mathrm{p}}(\beta)$ after 30 iterations as a function of the load multiplier $\beta$ are shown in Figs 6(a) and (b), respectively. In Fig. 6(a), $\sigma_{y}^{*}$ decreases, reaching its minimum value of plastic admissibility on the plastic yield surface $F[\boldsymbol{\sigma}]=\mathrm{Tol}$ (for $T o l=0.01$ ) shown as a dashed line. The vectors $\sigma^{*}$ and $\sigma$ characterize the predictor and the admissible stress, respectively. In Fig. 6(b), the stored plastic strain is shown over the same load interval used in Fig. 6(a). Inside each load step, the plastic strain at first increases rapidly and then its growth decreases. In all cases, the curve asymptotically tends toward infinity.

Displacement diagrams for the nodes $1,2, \ldots, 8$, located along a sloping line with respect to the horizontal axis for various values of the load multiplier $\beta$ are shown in Fig. 7(a). These diagrams show that when the load multiplier is lower than $\beta=3$, the response is elastic as shown in Fig. 7(b). For load multipliers greater than $\beta=3$, the response becomes elasto-plastic and the bem-e located at the corner is subjected to large plastic strains, whereas the other bem-es store the plastic strains for high values of the load multiplier up to close to the collapse of the plate.

It should be noted that: 


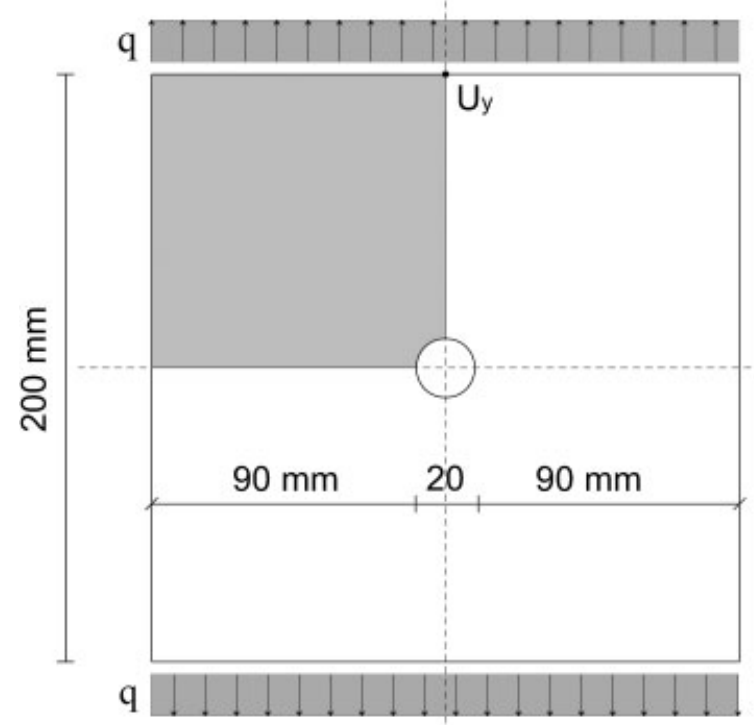

a)

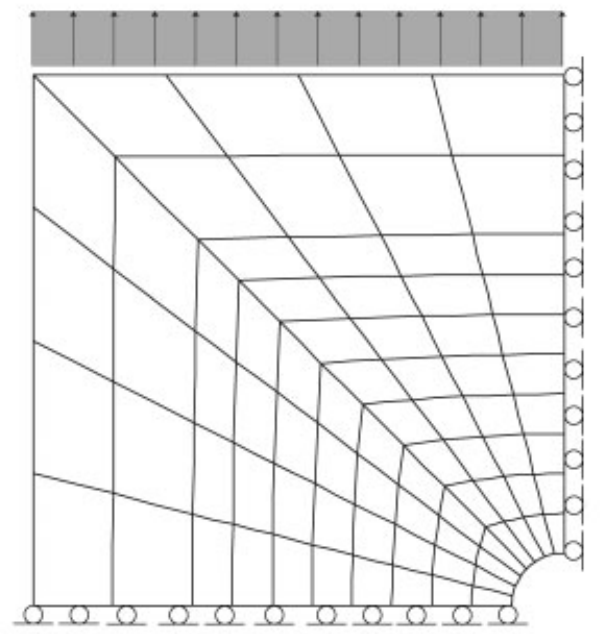

c)

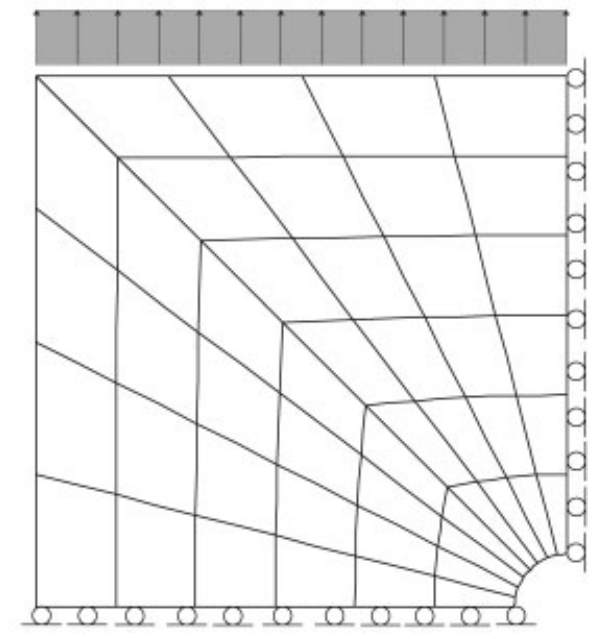

b)

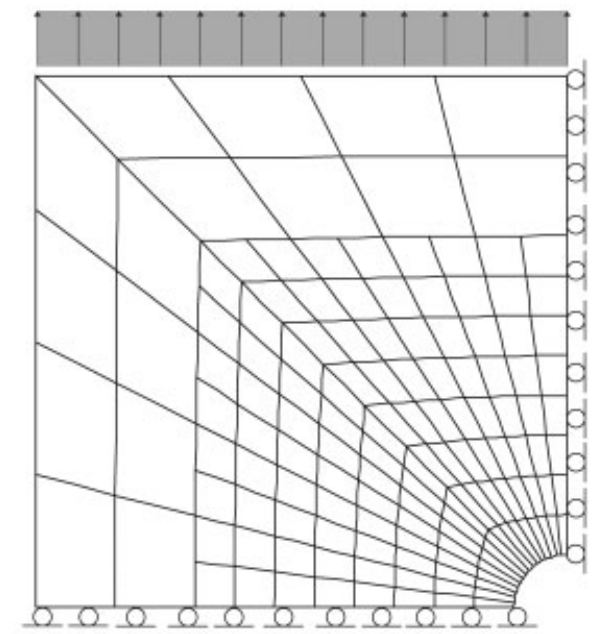

d)

Fig. 3 Plate with circular hole: (a) geometric description, (b) first, (c) second, and (d) third meshs

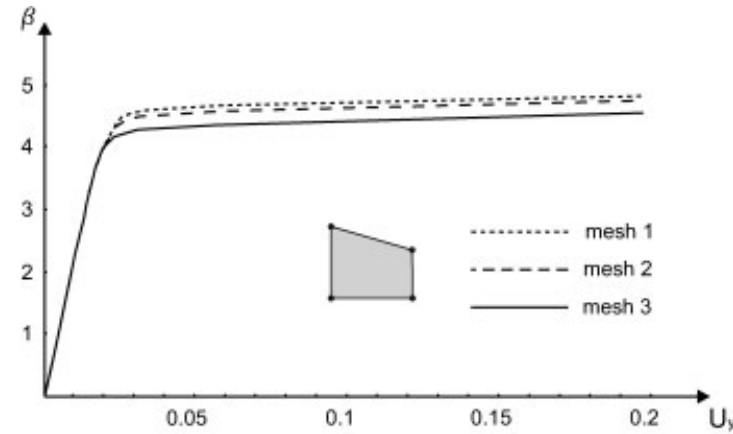

a)

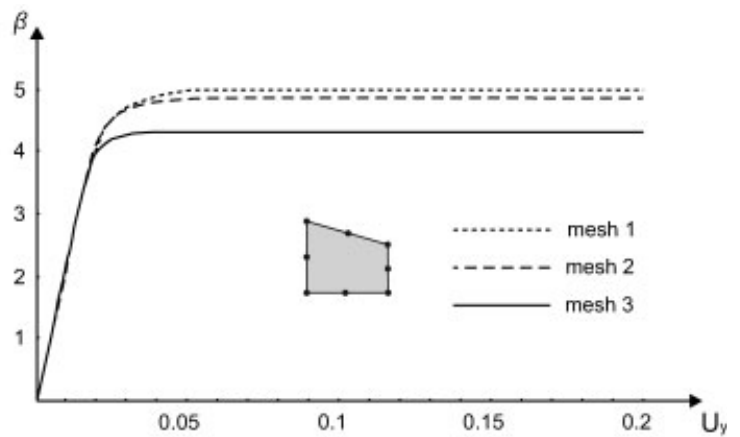

b)

Fig. 4 Load-displacement curves: (a) four-node bem-e after 300 iterations and (b) eight-node bem-e after 30 iterations 


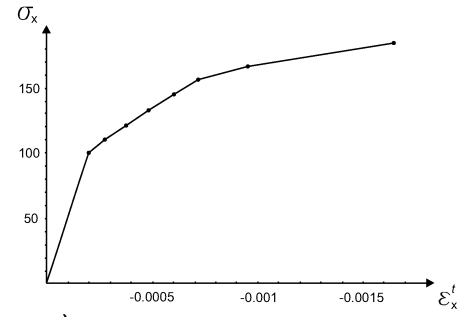

a)

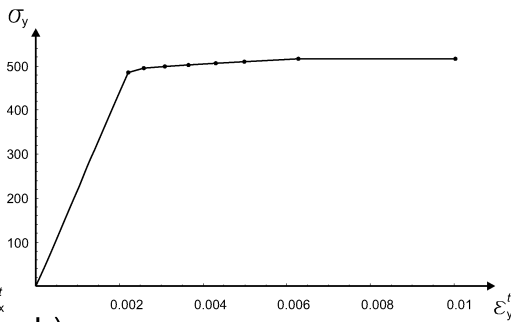

b)

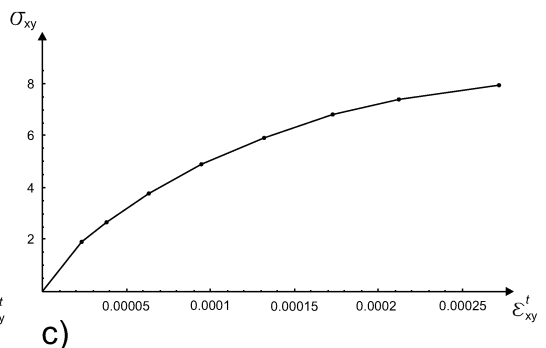

c)

Fig. 5 The constitutive behaviour in (a) the $x$-, (b) the $y$-, and (c) the $x y$-directions

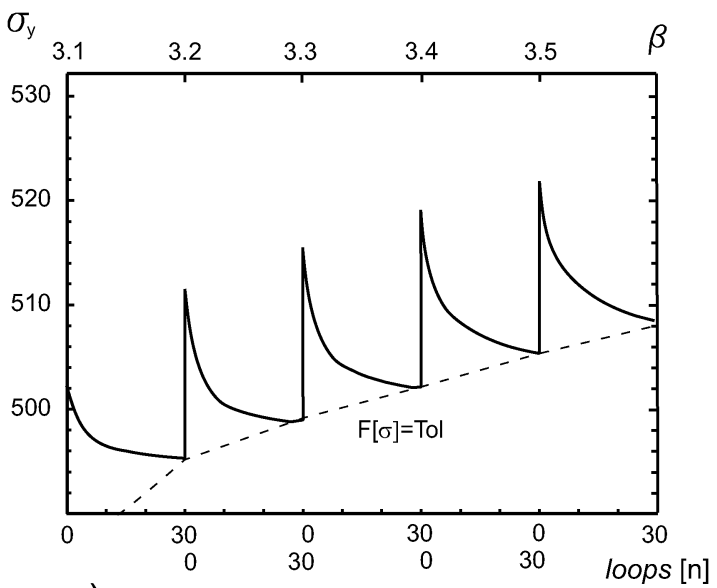

a)

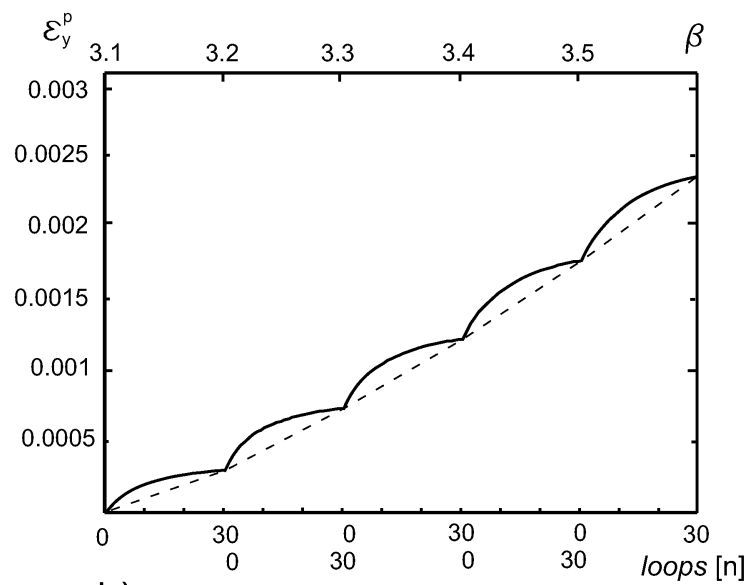

b)

Fig. 6 (a) The stress and (b) the plastic strain at the Gauss point for the bem-e located at the corner of the hole

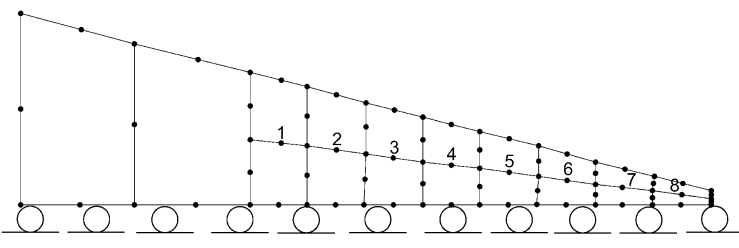

a)

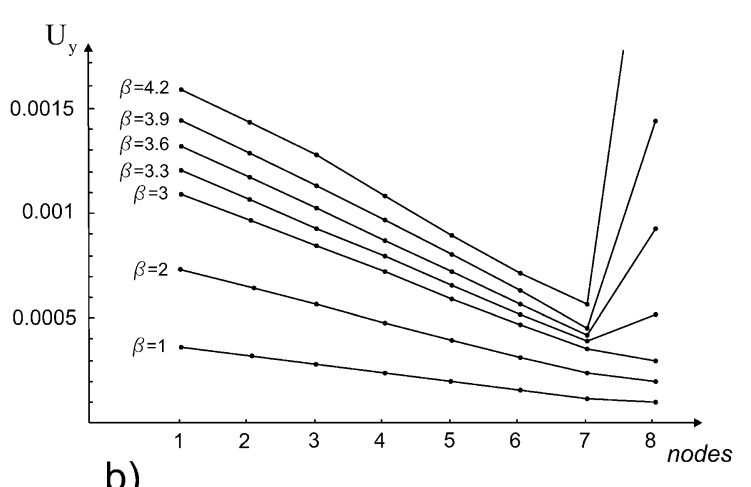

b)

Fig. 7 Plate with circular hole: (a) the horizontal roller constraint and (b) the vertical displacements

(a) for the eight-node bem-e case, analyses performed for values of the load multiplier near to the collapse point became numerically unstable;

(b) for the four-node bem-e case, the response was more stable, but this is at the cost of a very high computational effort;

(c) for both cases, the load multipliers, which determines the onset of the plastic phase, are similar.

\subsection{Notched specimen}

In the second example, a notched specimen subjected to tensile load $q=5 \mathrm{MPa}$ amplified by $\beta$, is analysed. The material parameters are a Young's modulus $E=20000$, a Poisson's ratio $v=0.3$, and a uniaxial yield value of $\sigma_{\mathrm{y}}=30 \mathrm{MPa}$. The geometrical data are given in Fig. 8(a). Again three different types of meshes were considered: 


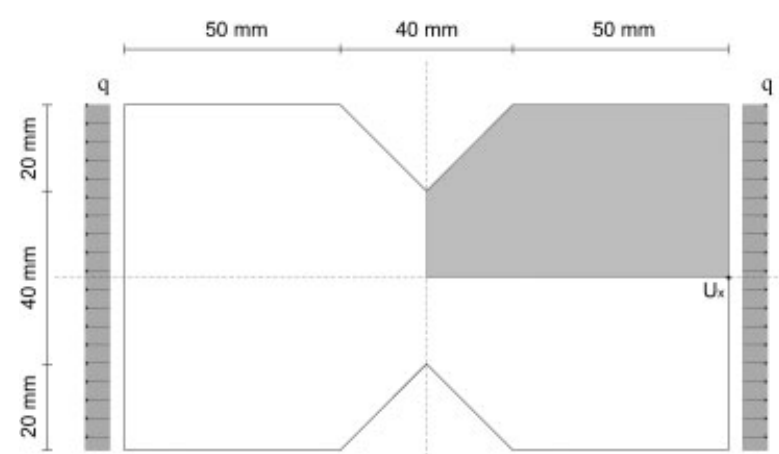

a)

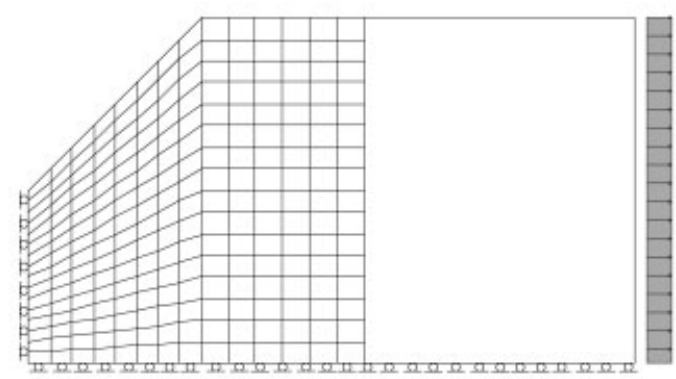

c)

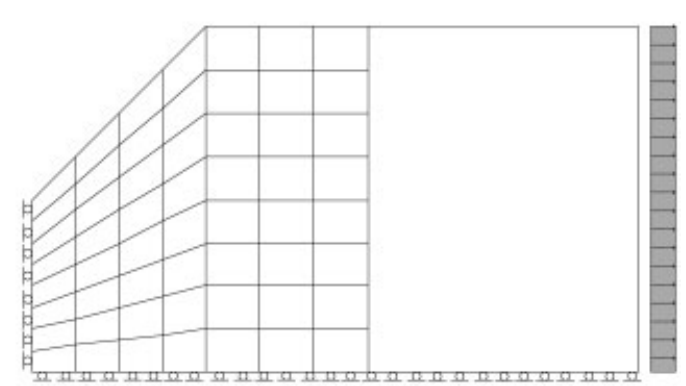

b)

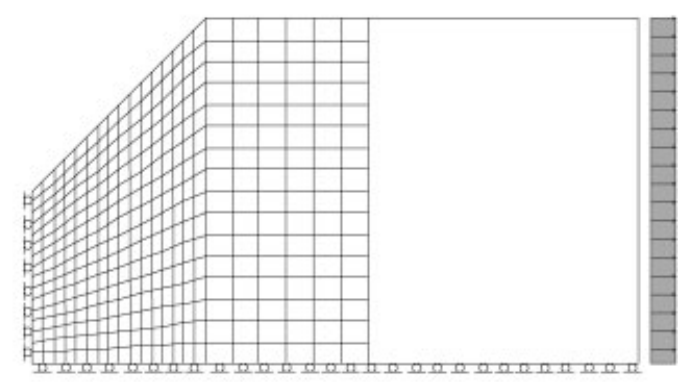

d)

Fig. 8 Notched specimen tests: (a) geometrical data, and (b) first, (c) second, and (d) third meshes

(a) the first mesh had 56 bem-es, see Fig. 8(b);

(b) the second mesh had 224 bem-es, see Fig. 8(c);

(c) the third mesh had 352 bem-es, see Fig. 8(d).

Two different elasto-plastic analyses were performed: first, with a four-node bem-e and a maximum of 300 iterations and second with an eight-node bem-e and 30 iterations. These numerical tests were performed with the aim of showing that the 8-node bem-e can accurately describe the perfectly plastic behaviour.

The load-displacement curves relating to the three meshes are shown for both the four-node bem-e and
300 iterations (Fig. 9(a)) and the eight-node bem-e and 30 iterations (Fig. 9(b)). The use of the fournode bem-e leads to very poor results in that it does not create a good elasto-plastic response, which is due its stiffer behaviour than for the eight-node case. The eight-node case resulted in an elastic-perfectlyplastic response of the structure, i.e. in a coherent manner with the adopted constitutive law.

Figure 10 displays contour maps of the equivalent plastic strains at collapse, calculated using the fournode bem-e and 300 iterations, for the three considered meshes.

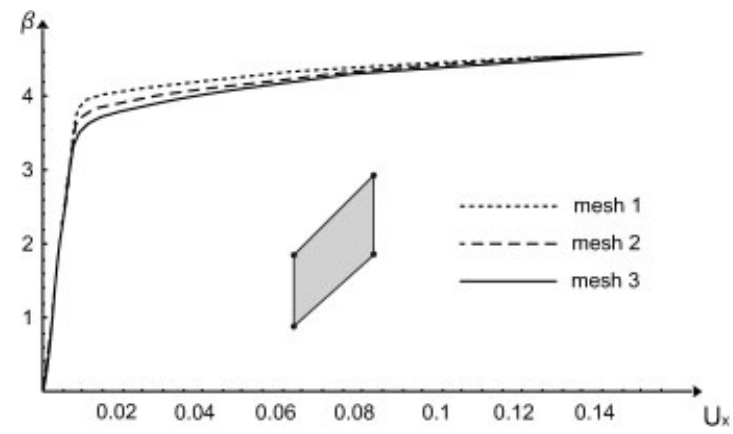

a)

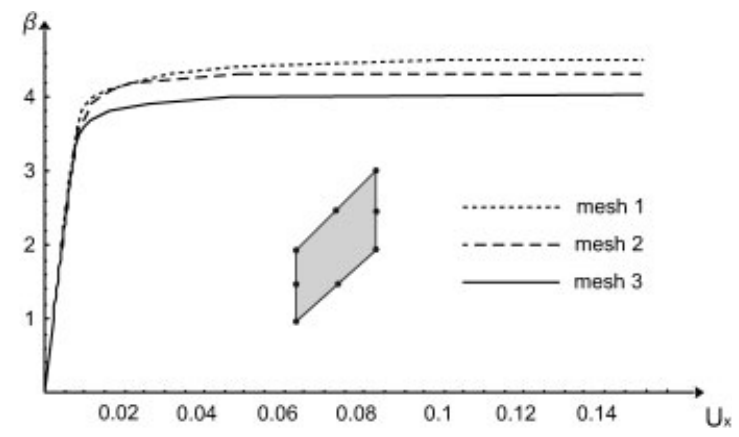

b)

Fig. 9 Load-displacement curves: (a) four-node bem-e after 300 iterations and (b) eight-node bem-e after 30 iterations 

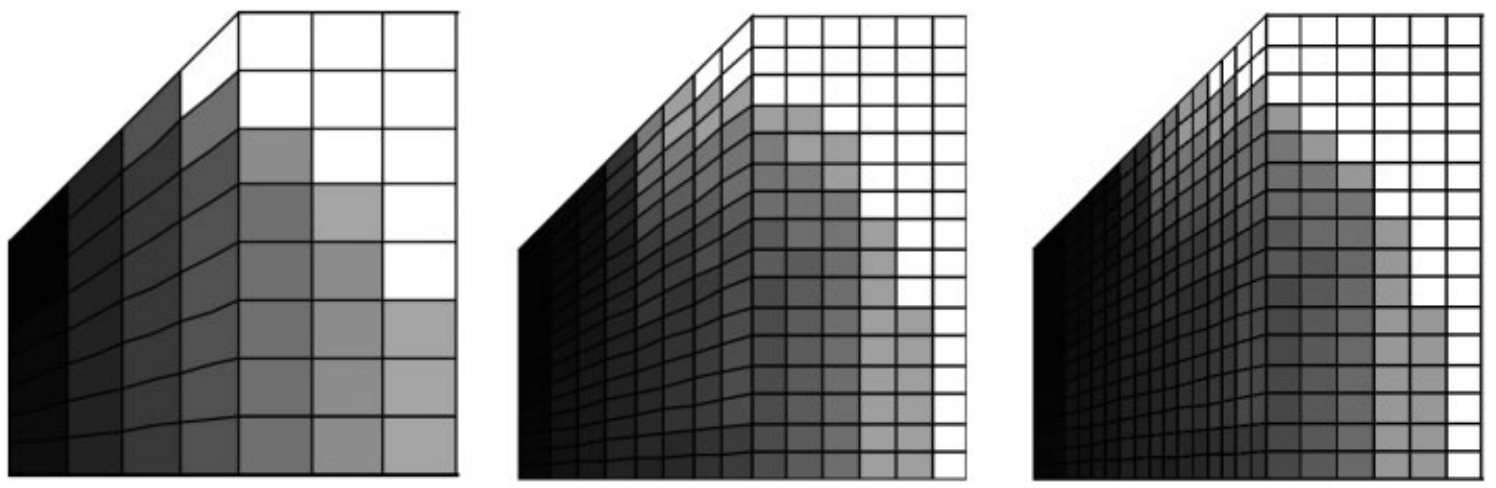

Fig. 10 Contour maps of the equivalent plastic strain at collapse

\subsection{Skew beam}

In the third example, a skew beam subjected to shear load $q=0.1 \mathrm{MPa}$ amplified by $\beta$ is analysed. The material parameters are a Young's modulus $E=20000$, a Poisson's ratio $v=0.3$, and a uniaxial yield value of $\sigma_{\mathrm{y}}=1.0 \mathrm{MPa}$. The geometrical data are given in Fig. 11(a). Again three different types of meshes were considered:

(a) the first mesh had 32 bem-es, see Fig. 11(b);

(b) the second mesh had 128 bem-es, see Fig. 11(c);

(c) the third mesh had 512 bem-es, see Fig. 11(d).

The elasto-plastic analyses were performed using an eight-node bem-e and 30 iterations.
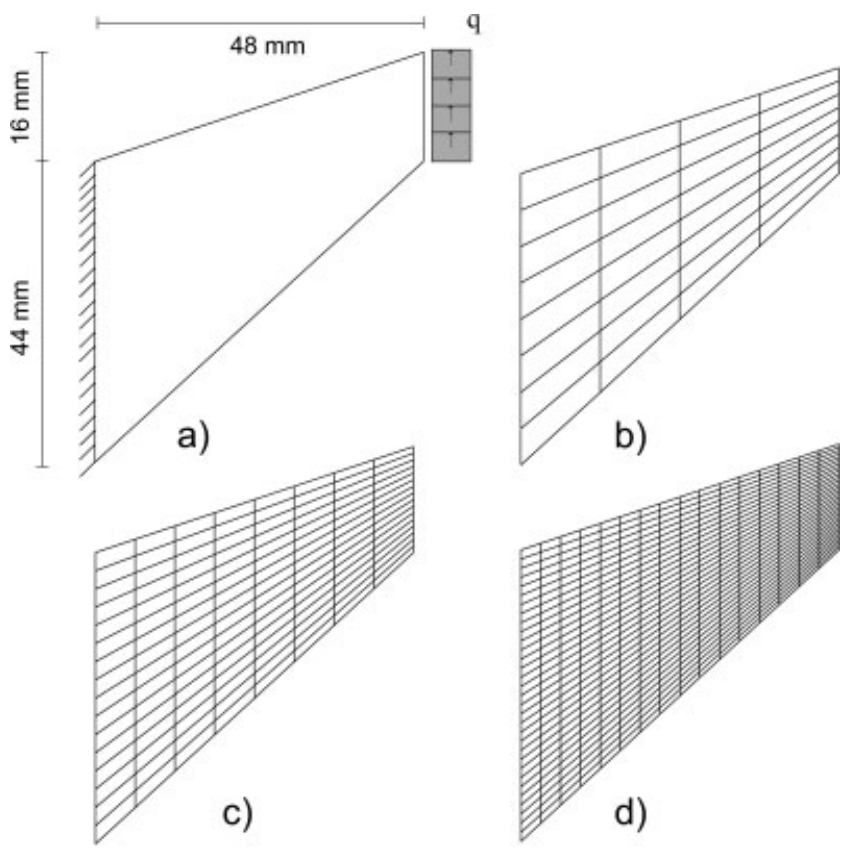

Fig. 11 Skew beam tests: (a) geometrical data, and (b) first, (c) second, and (d) third meshes
The load-displacement curves for the three meshes are shown in Fig. 12. The solution obtained by the present approach are compared with two FEM solutions: the first uses a standard element (Q4), the second a high performance element (Flex4) [19]. It can be seen from Fig. 12 that our results are very close to those obtained by FE methods; for example, Fig. 12(c) (third mesh) shows that the present solutions differ from Q4 and Flex4 solutions by about 0.49 and 1.37 per cent, respectively.

\section{CONCLUSIONS}

The computational aspects of a new strategy for the modelling of elasto-plastic effects that couples the SBEM with a return mapping scheme has been considered in this paper. Attention has been mainly focused on 2D continuous solids in order to allow the use of the elastic-perfectly-plastic von Mises' law, however, the proposed methodology is general enough for it to be applied to more complex plastic models, i.e. those with hardening, softening laws, etc. Elastic analyses were performed using the SBEM displacement approach and the advantage accrued by producing a model that only contains nodal kinematical unknowns of the interface boundaries has been demonstrated.

The solution was obtained by exploiting the elasto-plastic constitutive law, which involves elastic and plastic terms. A return mapping procedure, in which the computed elastic predictor is relaxed onto a yield surface, was utilized in the multidomain SBEM analysis. In detail, the return mapping algorithm shown in this paper was defined by using the extremal paths theory. In addition, the computational problems created by the presence of domain integrals and singular kernels were appropriately considered. The proposed procedure transforms the 


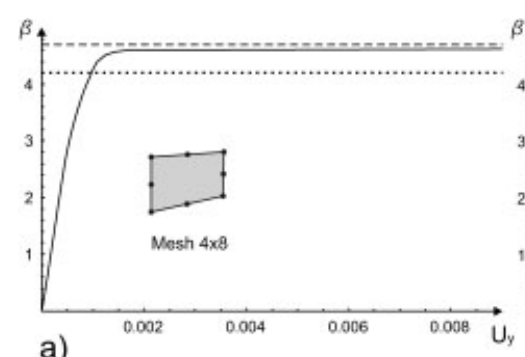

a)

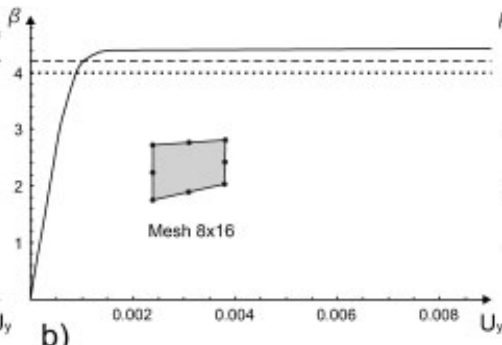

b)

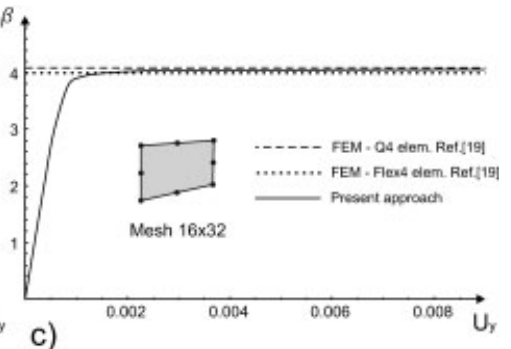

c)

Fig. 12 Load-displacement curves: (a) first, (b) second, and (c) third meshes

domain integrals into boundary integrals of each bem-e and the singularities present in the kernels are removed. Therefore, the SI of the traction is defined on the boundary through a limit operation.

This strategy, which utilizes only coefficients in closed form, has been coded into the software Karnak.sGbem [26] and is able to give solutions to plane structural systems with any geometry and subjected to both elastic and inelastic actions. Applications have been presented that show the effectiveness of the proposed procedure.

(C) Authors 2011

\section{REFERENCES}

1 Perez-Gavilan, J. J. and Aliabadi, M. H. Symmetric Galerkin BEM for Multi-connected bodies. Commun. Numer. Meth. Engng, 2001, 17, 761-770.

2 Perez-Gavilan, J. J. and Aliabadi, M. H. A symmetric Galerkin BEM for multi-connected bodies: a new approach. Engng. Anal. Bound. Elem., 2001, 25, 633-638.

3 Brebbia, C. A., Telles, J. C. F., and Wrobel, L. C. Boundary element techniques. Theory and application in engineering, 1984 (Springer, Berlin, Germany).

4 Aliabadi, M. H. The boundary element method. Applications in solids and structures, vol. 2, 2002 (Wiley, London, UK).

5 Telles, J. C. F. and Carrer, J. A. M. Implicit procedures for the solution of elastoplastic problems by boundary element method. Math. Comput. Model., 1991, 15, 303-311.

6 Banerjee, P. K. The boundary element method in engineering, 1994 (McGraw Hill, London, UK).

7 Bonnet, M. and Mukherjee, S. Implicit BEM formulations for usual and sensitivity problems in elastoplasticity using the consistent tangent operator concept. Int. J. Solids Struct., 1996, 33, 44614480.

8 Simo, J. C. and Taylor, R. L. Consistent tangent operators for rate-independent elastoplasticity. Comput. Meth. Appl. Mech. Engng, 1995, 48, 101-118.
9 Paulino, G. H. and Yong, L. Implicit consistent and continuum tangent operators in elastoplastic boundary element formulations. Comput. Meth. Appl. Mech. Engng, 2001, 190, 2157-2179.

10 Mallardo, V. and Alessandri, C. Arc-length procedures with BEM in physically nonlinear problems. Engng. Anal. Bound. Elem., 2004, 28, 547-559.

11 Maier, G. and Polizzotto, C. A Galerkin approach to boundary element elastoplastic analysis. Comput. Meth. Appl. Mech. Engng, 1987, 60, 175-194.

12 Polizzotto, C. An energy approach to the boundary element method, part I: elastic solids. Comput. Meth. Appl. Mech. Engng, 1988, 69, 167-184.

13 Polizzotto, C. An energy approach to the boundary element method, part II: elastic-plastic solids. Comput. Meth. Appl. Mech. Engng, 1988, 69, 263276.

14 Lie, S. T., Xu, K., and Liu, Q. Elastoplastic analysis using quasi-higher order symmetric Galerkin boundary element method. Engng Comput., 1999, 16, 871-891.

15 Panzeca, T., Salerno, M., and Terravecchia, S. Domain decomposition in the symmetric boundary element method analysis. Comput. Mech., 2002, 28, 191-201.

16 Panzeca, T., Cucco, F., and Terravecchia, S. Symmetric boundary element method versus finite element method. Comput. Meth. Appl. Mech. Engng, 2002, 191, 3347-3367.

17 Ortiz, M. and Simo, J. C. An analysis of a new class of integration algorithm for elastoplastic constitutive relations. Int. J. Numer. Meth. Engng, 1986, 23, 353-366.

18 Ortiz, M. and Martin, J. B. Symmetry-preserving return mapping algorithms and incrementally extremal paths: a unification of concepts. Int. J. Numer. Meth. Engng, 1989, 28, 1839-1853.

19 Bilotta, A. and Casciaro, R. A high-performance element for the analysis of 2D elastoplastic continua. Comput. Meth. Appl. Mech. Engng, 2007, 196, 818-828.

20 Ponter, A. R. S. and Martin, J. B. Some extremal properties and energy theorems for inelastic materials and their relationship to the deformation theory of plasticity. J. Mech. Phys. Solids, 1972, 20, 281-300.

21 Bui, H. D. Some remarks about the formulation of three-dimensional thermoelastoplastic problems 
by integral equation. Int. J. Solids Struct., 1978, 14, 935-939.

22 Gao, X. W. The radial integration method for evaluation of domain integrals with boundary-only discretization. Engng. Anal. Bound. Elem., 2002, 26, 905-916.

23 Panzeca, T., Terravecchia, S., and Zito, L. Computational aspects in 2D SBEM analysis with domain inelastic actions. Int. J. Numer. Meth. Engng, 2010, 82, 184-204.

24 Young, A. Improved numerical method for traction boundary integral equation by application of Stokes theorem. Int. J. Numer. Meth. Engng, 1997, 40, 3141-3161.

25 Wilde, A. J. and Aliabadi, M. H. Direct evaluation of boundary stresses in 3-D BEM of elastostatic. Commun. Numer. Meth. Engng, 1998, 14, 505-517.

26 Panzeca, T., Cucco, F., and Terravecchia, S. The program Karnak.sGbem, Release 2.1, 2002.

27 Panzeca, T., Fujita Yashima, H., and Salerno, M. Mathematical aspects and applications of the symmetric Galerkin boundary element method. In, (Eds Idelsohn, S. R., Onate, E., and Dvorkin, E. N.), 1988 (Buenos Aires, Argentina).

28 Panzeca, T., Fujita Yashima, H., and Salerno, M. Direct stiffness matrices of BEs in the Galerkin BEM formulation. Eur. J. Mech. A, Solids, 2000, 20, 277-298.

29 Terravecchia, S. Closed form coefficients in the symmetric boundary element approach. Engng. Anal. Bound. Elem., 2006, 30, 479-488.

30 Gao, X. W. Boundary element analysis in thermoelasticity with and without internal cells. Int. J. Numer. Meth. Engng, 2003, 57, 975-990.

31 Gao, X. W. and Davies, T. G. An effective boundary element algorithm for $2 \mathrm{D}$ and $3 \mathrm{D}$ elastoplastic problem. Int. J. Solids Struct., 2000, 37, 4987-5008.

\section{APPENDIX}

In this appendix it is shown that the domain integrals containing the body forces $\overline{\boldsymbol{b}}$ (equations (29), (40b), and (41)) can be transformed into boundary integrals, in order to provide an easily generalizable procedure

$$
\begin{gathered}
\boldsymbol{u}[\overline{\boldsymbol{b}}]=\int_{\Omega} \mathbf{G}_{u u} \overline{\boldsymbol{b}} \mathrm{d} \Omega \\
\boldsymbol{t}[\overline{\boldsymbol{b}}]=\int_{\Omega} \mathbf{G}_{t u} \overline{\boldsymbol{b}} \mathrm{d} \Omega
\end{gathered}
$$

$$
\boldsymbol{\sigma}[\overline{\boldsymbol{b}}]=\int_{\Omega} \mathbf{G}_{\sigma u} \overline{\boldsymbol{b}} \mathrm{d} \Omega
$$

Referring the generic bem-e subjected to constant distribution body forces $\overline{\boldsymbol{b}}$, consider the coefficient in equation (49b), which is transferred onto the boundary by a double transformation into polar coordinates and vice versa

$$
\begin{gathered}
\int_{\Omega} \mathbf{G}_{t u} \mathrm{~d} \Omega \times \overline{\boldsymbol{b}}=\int_{0}^{2 \pi} \int_{0}^{R} \frac{\boldsymbol{\Psi}_{t u}(\varphi)}{r} r \mathrm{~d} r \mathrm{~d} \varphi \cdot \overline{\boldsymbol{b}} \\
=\int_{0}^{2 \pi} \frac{\boldsymbol{\Psi}_{t u}(\varphi)}{R} R^{2} \mathrm{~d} \varphi \times \overline{\boldsymbol{b}}=\int_{\Gamma} \mathbf{G}_{t u} \boldsymbol{n}^{\mathrm{T}} \boldsymbol{r} \mathrm{d} \Gamma \cdot \overline{\boldsymbol{b}}
\end{gathered}
$$

when $R^{2} \mathrm{~d} \varphi=\boldsymbol{n}^{\mathrm{T}} \boldsymbol{r} \mathrm{d} \Gamma$.

This strategy allows the computation of the tractions due to body forces transferred onto the boundary

$$
\begin{aligned}
\boldsymbol{t}[\overline{\boldsymbol{b}}] & =\int_{\Omega} \mathbf{G}_{t u} \overline{\boldsymbol{b}} \mathrm{d} \Omega \\
& =\int_{\Gamma} \tilde{\mathbf{G}}_{t u} \overline{\boldsymbol{b}} \mathrm{d} \Gamma \quad \text { where } \quad \tilde{\mathbf{G}}_{t u}=\mathbf{G}_{t u}\left(\boldsymbol{n}^{\mathrm{T}} \boldsymbol{r}\right)
\end{aligned}
$$

A similar strategy may be used for the displacements

$$
\boldsymbol{u}[\overline{\boldsymbol{b}}]=\int_{\Omega} \mathbf{G}_{u u} \overline{\boldsymbol{b}} \mathrm{d} \Omega=\int_{\Gamma} \tilde{\mathbf{G}}_{u u} \overline{\boldsymbol{b}} \mathrm{d} \Gamma
$$

where $\tilde{\mathbf{G}}_{u u}=1 / 2\left[\mathrm{G}_{u u}+q \mathrm{I}\right]\left(\mathrm{n}^{\mathrm{T}} \mathrm{r}\right)$

I being the identity matrix and $q=(3-4 v)(1+v) /$ $(8 E(1-v) \pi)$.

This strategy can also be used for the stresses

$$
\boldsymbol{\sigma}[\overline{\boldsymbol{b}}]=\int_{\Omega} \mathbf{G}_{\sigma u} \overline{\boldsymbol{b}} \mathrm{d} \Omega=\int_{\Gamma} \tilde{\mathbf{G}}_{\sigma u} \overline{\boldsymbol{b}} \mathrm{d} \Gamma
$$

where $\tilde{\mathbf{G}}_{\sigma u}=\mathrm{G}_{\sigma u}\left(\mathrm{n}^{\mathrm{T}} \mathrm{r}\right)$ 